\title{
Non-Markovian dynamics for a free quantum particle subject to spontaneous collapse in space: general solution and main properties
}

\author{
Angelo Bassi* and Luca Ferialdit \\ Dipartimento di Fisica Teorica, Università di Trieste, Strada Costiera 11, 34014 Trieste, Italy.
}

Istituto Nazionale di Fisica Nucleare, Sezione di Trieste, Strada Costiera 11, 34014 Trieste, Italy.

\begin{abstract}
We analyze the non-Markovian dynamics of a quantum system subject to spontaneous collapse in space. After having proved, under suitable conditions, the separation of the center-of-mass and relative motions, we focus our analysis on the time evolution of the center of mass of an isolated system (free particle case). We compute the explicit expression of the Green's function, for a generic Gaussian noise, and analyze in detail the case of an exponential correlation function. We study the time evolution of average quantities, such as the mean position, momentum and energy. We eventually specialize to the case of Gaussian wave functions, and prove that all basic facts about collapse models, which are known to be true in the white noise case, hold also in the more general case of non-Markovian dynamics.
\end{abstract}

PACS numbers: 03.65.Ta, 02.50.Ey, 05.40.-a

\section{INTRODUCTION}

Among the several models of spontaneous wave function collapse which has been considered so far, the so-called QMUPL (Quantum Mechanics with Universal Position Localization) model is particularly interesting, being it a very good compromise between mathematical simplicity and

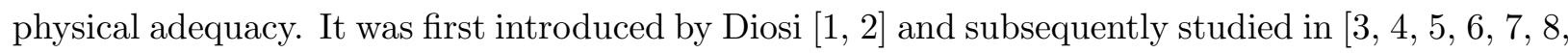
$9,10,11]$, both from the mathematical as well as physical point of view. It is particularly relevant because it is the simplest model describing the evolution of the wave function of a system of $N$ distinguishable particles, subject to a spontaneous collapse in space; as such, it can be analyzed in great mathematical detail. The model is defined by the following stochastic differential equation $(\mathrm{SDE})$ :

$$
d \psi_{t}(\{x\})=\left[-\frac{i}{\hbar} H d t+\sum_{n=1}^{N} \sqrt{\lambda_{n}}\left(q_{n}-\left\langle q_{n}\right\rangle_{t}\right) d W_{n, t}-\frac{1}{2} \sum_{n=1}^{N} \lambda_{n}\left(q_{n}-\left\langle q_{n}\right\rangle_{t}\right)^{2} d t\right] \psi_{t}(\{x\})
$$

\footnotetext{
*Electronic address: bassi@ts.infn.it

${ }^{\dagger}$ Electronic address: ferialdi@ts.infn.it
} 
where the symbol $\{x\} \equiv x_{1}, x_{2}, \ldots x_{n}$ denotes the coordinates of the $N$ particles (for simplicity, we will work in one spatial dimension). The operator $H$ is the standard quantum hamiltonian of the composite system; $q_{n}$ is the position operator associated to the $n$-th particle and $\left\langle q_{n}\right\rangle_{t} \equiv\left\langle\psi_{t}\left|q_{n}\right| \psi_{t}\right\rangle$ denotes the quantum expectation value of $q_{n}$. The stochastic processes $W_{n, t}$ are $N$ independent standard Wiener processes defined on a probability space $(\Omega, \mathcal{F}, \mathbb{P})$, and the parameters $\lambda_{n}$ are $N$ positive coupling constants which is convenient to take proportional to the mass of the particle according to the formula [9]:

$$
\lambda_{n}=\frac{m_{n}}{m_{0}} \lambda_{0},
$$

where $m_{n}$ is the mass of the $n$-th particle, $m_{0}$ is a reference mass which, at the non relativistic level, is reasonable to take equal to the mass of a nucleon $\left(m_{0} \simeq 1.67 \times 10^{-27} \mathrm{Kg}\right)$, while $\lambda_{0}$ is the only true new parameter of the model, whose value sets the strength of the collapse mechanism.

The numerical value of $\lambda_{0}$ has to be chosen in such a way that: i) the model reproduces quantum mechanical predictions for microscopic systems; ii) it rapidly induces the collapse of the wave function describing the center of mass of a macroscopic object. In the literature, two quite different values for $\lambda_{0}$ have been proposed, the first by Ghirardi, Rimini, and Weber [12], and the second by Adler [13]:

$$
\begin{aligned}
& \lambda_{0}^{\mathrm{GRW}} \simeq 5.00 \times 10^{-3} \mathrm{~m}^{-2} \mathrm{sec}^{-1} \\
& \lambda_{0}^{\text {Adler }} \simeq 1.12 \times 10^{6 \pm 2} \mathrm{~m}^{-2} \mathrm{sec}^{-1} .
\end{aligned}
$$

GRW's choice is motivated by the requirement that superpositions of order $6.02 \times 10^{23}$ nucleons, displaced by a distance of at least $r_{C}=10^{-5} \mathrm{~cm}$, be suppressed within $10^{-3}$ sec. Adler's choice instead is motivated by the requirement that the collapse occurs already at the level of latent image formation. More specifically, GRW set $\lambda_{\mathrm{GRW}} \simeq 10^{-16} \mathrm{sec}^{-1}$, where $\lambda_{\mathrm{GRW}}$ is the collapse rate of the GRW model [12]; Adler instead set $\gamma_{\mathrm{CSL}} \simeq 2 \times 10^{-21 \pm 2} \mathrm{~cm}^{3} \mathrm{sec}^{-1}$ [13], where $\gamma_{\mathrm{CSL}}$ is the noise-strength coupling constant of the CSL model; the relation between $\lambda_{0}$ of our model and $\lambda_{\text {GRW }}$ and $\gamma_{\mathrm{CSL}}$ is $\left[9\right.$, 14] $: \lambda_{0}=\alpha \lambda_{\mathrm{GRW}} / 2=\alpha^{5 / 2} \gamma_{\mathrm{CSL}} / 16 \pi^{3 / 2}$, with $\alpha=1 / r_{C}^{2}$.

Eq. (1) is manifestly not linear, which makes it difficult to analyze, in particular it makes it hard to find its solutions. The way to circumvent this obstacle is to linearize the equation, according to the following prescription [8]. Consider the linear SDE:

$$
d \phi_{t}(\{x\})=\left[-\frac{i}{\hbar} H d t+\sum_{n=1}^{N} \sqrt{\lambda_{n}} q_{n} d \xi_{n, t}-\frac{1}{2} \sum_{n=1}^{N} \lambda_{n} q_{n}^{2} d t\right] \phi_{t}(\{x\}),
$$


where the stochastic processes $\xi_{n, t}$ are $N$ independent standard Wiener processes with respect to a new measure $\mathbb{Q}$. It can be shown that $\left\|\psi_{t}\right\|^{2}$ is a martingale, which can be used to generate a new measure from a given one: the measure $\mathbb{Q}$ introduced here above is chosen in such a way that $\left\|\psi_{t}\right\|^{2}$ is the Radon-Nikodyn derivative of $\mathbb{P}$ with respect to $\mathbb{Q}$, i.e.: $d \mathbb{P}=\left\|\phi_{t}\right\|^{2} d \mathbb{Q}$. Moreover, Girsanov's theorem [15] states that the two sets of Wiener processes $\left\{W_{n, t}\right\}$ and $\left\{\xi_{n, t}\right\}$ are related as follows: $d W_{n, t}=d \xi_{n, t}-2 \sqrt{\lambda}\left\langle q_{n}\right\rangle_{t} d t$.

Given these ingredients, it is easy to relate the solutions of Eq. (15) to those of Eq. (1): given a solution $\phi_{t}$ of Eq. (5), one first considers the normalized state $\psi_{t}=\phi_{t} /\left\|\phi_{t}\right\|$, and then replaces the noises $\left\{\xi_{n, t}\right\}$ with $\left\{W_{n, t}\right\}$ according to the formula given above. It is not difficult to show that the wave function so obtained solves Eq. (11). Further details can be found in [8].

The aim of this paper is to analyze the generalization of the QMUPL model to non-Markovian Gaussian random processes. As discussed in [16, 17, 18], the generalization of the linear Eq. (5) to the non-Markovian case takes the form:

$$
\frac{d}{d t} \phi_{t}(\{x\})=\left[-\frac{i}{\hbar} H+\sum_{n=1}^{N} \sqrt{\lambda_{n}} q_{n} w_{n}(t)-2 \sum_{n, m=1}^{N} \sqrt{\lambda_{n}} q_{n} \int_{0}^{t} d s D_{n m}(t, s) \frac{\delta}{\delta w_{m}(s)}\right] \phi_{t}(\{x\}),
$$

where the noises $w_{n}(t)$ are now supposed to be Gaussian random processes whose mean and correlation function, expressed with respect to the measure $\mathbb{Q}$, are equal to:

$$
\mathbb{E}_{\mathbb{Q}}\left[w_{n}(t)\right]=0, \quad \mathbb{E}_{\mathbb{Q}}\left[w_{n}(t) w_{m}(s)\right]=D_{n m}(t, s) .
$$

The correlation function is assumed to be real, symmetric and positive-semidefinite [19].

Like in the white-noise case, Eq. (6) does not preserve the norm of the wave function. Accordingly, we assume that the physical states are the normalized states:

$$
\psi_{t}(\{x\}) \equiv \frac{\phi_{t}(\{x\})}{\left\|\phi_{t}\right\|}
$$

moreover, we assume that the physical probability measure is $\mathbb{P}$, which is related to the measure $\mathbb{Q}$, by means of which the statistical properties of the noises $w_{n}(t)$ have been defined, according to the formula:

$$
d \mathbb{P} \equiv\left\|\phi_{t}\right\|^{2} d \mathbb{Q}
$$

Measurable quantities are given by expressions of the form: $\mathbb{E}_{\mathbb{P}}\left[\left\langle\psi_{t}|O| \psi_{t}\right\rangle\right]$, where $O$ is a suitable self adjoint operator. According to relation (9), the following equality holds true:

$$
\mathbb{E}_{\mathbb{P}}\left[\left\langle\psi_{t}|O| \psi_{t}\right\rangle\right] \equiv \mathbb{E}_{\mathbb{P}}\left[\left\langle\phi_{t}|O| \phi_{t}\right\rangle\right]
$$


We then have the following useful results: physical properties can be computed directly from the solution of the linear equation (6), if one averages with respect to the measure $\mathbb{Q}$, instead of the physical measure $\mathbb{P}$. For this reason, in the following we will focus our attention only on Eq. (6) , without trying to investigate the form of the corresponding non-linear but norm-preserving equation. This will be the subject of future analysis.

The goal of the paper is to unfold the dynamics described by Eq. (6) $)$. We will focus our attention on the free particle case, which can be solved exactly. The results can be generalized to the case of harmonic oscillators, and in general to any equation of the type (6), containing expressions which are at most quadratic in the operators $q$ and $p$. For other type of systems, perturbation techniques can be employed [20, 21, 22]. We list the main results we have obtained.

Result 1 (Sec. 【I). Under the conditions:

$$
\sum_{m=1}^{N}\left[\sqrt{\lambda_{m}} D_{m n}(t, s)-\sqrt{\frac{\lambda_{n} \lambda_{m}}{\lambda_{N}}} D_{m N}(t, s)\right]=0, \quad n=1,2, \ldots N-1,
$$

the center-of-mass motion and the relative motion decouple. In particular, when $D_{n m}(t, s)=$ $\delta_{n m} D(t, s)$, then the two equations for the center-of-mass wave function $\phi_{t}^{\mathrm{cm}}$ and relative-motion wave function $\phi_{t}^{\text {rel }}$ are:

$$
\begin{gathered}
\frac{d}{d t} \phi_{t}^{\mathrm{cm}}=\left[-\frac{i}{\hbar} H_{\mathrm{cm}}+\sqrt{\lambda} Q w(t)-2 \sqrt{\lambda} Q \int_{0}^{t} d s D(t, s) \frac{\delta}{\delta w(s)}\right] \phi_{t}^{\mathrm{cm}}, \\
\frac{d}{d t} \phi_{t}^{\mathrm{rel}}=\left[-\frac{i}{\hbar} H_{\mathrm{rel}}+\sum_{n=1}^{N} \sqrt{\bar{\lambda}_{n}} \bar{q}_{n} \bar{w}_{n}(t)-2 \sum_{n, m=1}^{N-1} \sqrt{\bar{\lambda}_{n}} \bar{q}_{n} C_{n m} \int_{0}^{t} d s D(t, s) \frac{\delta}{\delta \bar{w}_{m}(s)}\right] \phi_{t}^{\mathrm{rel}},
\end{gathered}
$$

where $w(t)$ and $\lambda$ are defined in (32) and (39), and $\bar{w}_{n}(t), \bar{\lambda}_{n}$ are defined in (45) and (46).

RESUlt 2 (Sec. III). The Green's function associated to a free particle of mass $m\left(H=p^{2} / 2 m\right)$ is:

$$
G\left(x, t ; x_{0}, 0\right)=\sqrt{\frac{m}{2 i \pi \hbar t u(t)}} \exp \left[-\frac{i m}{2 \hbar}\left(x_{0} z^{\prime}(0)-x z^{\prime}(t)\right)+\frac{\sqrt{\lambda}}{2} \int_{0}^{t} d s w(s) z(s)\right],
$$

where $z(t)$ solves Eq. (77), with boundary conditions: $z(0)=x_{0}, z(t)=x$, while $u(t)$ is defined in Eq. (82). In particular, under the condition $D(t, s)=D(|t-s|)$, which represents time translation invariance of the noise $w(t)$, the Green's function (14) has the simpler structure:

$$
G\left(x, t ; x_{0}, 0\right)=\sqrt{\frac{m}{2 i \pi \hbar t u(t)}} \exp \left[-\mathcal{A}_{t}\left(x_{0}^{2}+x^{2}\right)+\mathcal{B}_{t} x_{0} x+\mathcal{C}_{t} x_{0}+\mathcal{D}_{t} x+\mathcal{E}_{t}\right],
$$

where:

$$
\mathcal{A}_{t}=k f_{t}^{\prime}(0), \quad \mathcal{B}_{t}=2 k f_{t}^{\prime}(t), \quad k:=\frac{i \hbar}{2 m},
$$


are deterministic coefficients, while:

$$
\begin{aligned}
\mathcal{C}_{t} & =-k h_{t}^{\prime}(0)+\frac{\sqrt{\lambda}}{2} \int_{0}^{t} d l w(l) f_{t}(l) \\
\mathcal{D}_{t} & =k h_{t}^{\prime}(t)+\frac{\sqrt{\lambda}}{2} \int_{0}^{t} d l w(l) f_{t}(t-l) \\
\mathcal{E}_{t} & =\frac{\sqrt{\lambda}}{2} \int_{0}^{t} d l w(l) h_{t}(l)
\end{aligned}
$$

are random coefficients. The function $f(s)$ is solution of Eq. (84), with boundary conditions $f(0)=1, f(t)=0$, while $h(s)$ is defined in Eq. (98). As we see, in the non-Markovian time translation invariant case the free-particle propagator has the same structure as in the white noise case.

RESUlt 3 (Sec. IV). Using the Green's function (14), one can rewrite the non-Markovian SDE for a free quantum particle in a simpler way, replacing the functional derivative with combinations of the position and momentum operators. The collapsing equation takes the form:

$$
\frac{d}{d t} \phi_{t}(x)=\left[-\frac{i}{\hbar} \frac{p^{2}}{2 m}+\sqrt{\lambda} q w(t)-2 \lambda q \int_{0}^{t} d s D(t, s)\left(q a_{t}(s)+p b_{t}(s)+c_{t}(s)\right)\right] \phi_{t}(x),
$$

where $a_{t}(s), b_{t}(s), c_{t}(s)$ are defined in (107)-(109). This result agrees with the Ansatz first introduced in Sec. 4.3 of Ref. [17].

RESUlT 4 (Secs. V] and VI). In the white noise limit, one recovers well-known results in literature. More interestingly, we have solved explicitly the case of an exponential correlation function:

$$
D(t, s)=\frac{\gamma}{2} e^{-\gamma|t-s|}
$$

in which case the functions $f(s)$ and $h(s)$, through which the coefficients $\mathcal{A}_{t}-\mathcal{E}_{t}$ of the Green's function can be computed, are:

$$
f(s)=\frac{\sum_{k}\left[r_{t}^{k} \sinh v_{k}(t-s)+u_{t}^{k} \cosh v_{k}(t-s)-u_{s}^{k}\right]}{\sum_{k}\left[2 c+r_{t}^{k} \sinh v_{k} t+u_{t}^{k} \cosh v_{k} t\right]}
$$

while $h(s)$ is given by (98) with

$$
\begin{aligned}
h^{\mathrm{P}}(s) & =-\frac{i \sqrt{\lambda} \hbar}{m} \int_{0}^{s} \bar{f}_{s}(l)\left(w^{\prime \prime}(l)-\gamma^{2} w(l)\right) d l, \\
\bar{f}_{s}(l) & =-\frac{1}{\zeta} \sum_{k}(-1)^{k} \frac{\sinh v_{k}(s-l)}{v_{k}} .
\end{aligned}
$$

RESUlt 5 (Sec.VII). We have proven the so called imaginary noise trick, which we use to compute the average value of physical quantities, in particular the average position $q$, average momentum 
$p$, and average kinetic energy $H_{0}=p^{2} / 2 m$ :

$$
\begin{aligned}
\frac{d}{d t} \mathbb{E}_{\mathbb{Q}}\left[\langle q\rangle_{t}\right] & =\frac{1}{m} \mathbb{E}_{\mathbb{Q}}\left[\langle p\rangle_{t}\right], \\
\frac{d}{d t} \mathbb{E}_{\mathbb{Q}}\left[\langle p\rangle_{t}\right] & =0, \\
\frac{d}{d t} \mathbb{E}_{\mathbb{Q}}\left[\left\langle H_{0}\right\rangle_{t}\right] & =\frac{\lambda \hbar^{2}}{m} \int_{0}^{t} d s D(t, s) .
\end{aligned}
$$

The above equation prove that a free quantum particle moves, in the average, like a classical particle, but its kinetic energy is not conserved, not even in the average. This is an expected feature of the model [21].

RESUlt 6 (Sec. VIII). We have studied the time evolution of Gaussian wave functions, analyzing in particular the time evolution of the spread in position, and how the fluctuations around the average of both the mean position and mean momentum scale with the mass of the particle. In all cases, we have recovered the results which hold true for the white noise models.

Before concluding this introductory section, two comments are at order. First, it may seem rather remarkable that we have been able to compute the Green's function associated to a nonMarkovian equation. In fact, dynamics with memory terms in general do now allow for solutions expressed in terms of a Green's function, whose nature is strictly linked to the Markovian character of the evolution. The fact that in our case we have been able to compute the Green's function appears less surprising if one looks at the way in which Eq. (6) has been derived: originally, the non-Markovian evolution was presented only in terms of a propagator (see the pioneering works of Feynman and Vernon on decoherence [23] and of P. Pearle [24, 25] on collapse models) and only afterwards a non-Markovian equation was devised, having that propagator as the associated Green's function [16, 26].

Second, it may appear odd that the integration interval in Eq. (6) starts from 0 and not from $-\infty$, as required by a truly non-Markovian dynamics. Here we are implicitly making the assumption that the state at time $s=0$ suffices to compute the subsequent evolution, without any need to know also the previous history of the system. This assumption, which actually is an approximation if the equation is taken as a fundamental equation, is based on the following argument. We assume that before $s=0$ the system had enough time to reach some equilibrium state, which does not depend on the way it has been reached; this is the physical argument behind the idea that the history prior to $s=0$ is unimportant. Then, at time $s=0$ a sudden change in the system occurs-e.g., due to a measurement-like interaction: Eq. (마) describes how the system evolves thereafter. 


\section{SEPARATION OF THE CENTER-OF-MASS AND RELATIVE DEGREES OF FREEDOM}

Let us suppose that the total Hamiltonian $H$ of Eq. (6) separates in a term referring only to the center of mass and one referring to the relative degrees of freedom: $H=H_{\mathrm{cm}}+H_{\mathrm{rel}}$. In this section we analyze under which conditions the dynamics of the two types of motion decouple from each other. To this end, let us introduce the center of mass position operator $Q$ and the relative position operators $\tilde{q}_{n}$ defined as follows:

$$
\begin{aligned}
Q & \equiv \frac{1}{M} \sum_{n=1}^{N} m_{n} q_{n}, \quad M \equiv \sum_{n=1}^{N} m_{n}, \\
\tilde{q}_{n} & \equiv q_{n}-Q .
\end{aligned}
$$

We will choose $Q$ and $\tilde{q}_{1}, \tilde{q}_{2}, \ldots, \tilde{q}_{N-1}$ as a set of independent variables, while $\tilde{q}_{N}$ is given in terms of the other operators according to the formula:

$$
\tilde{q}_{N}=-\frac{1}{m_{N}} \sum_{n=1}^{N-1} m_{n} \tilde{q}_{n}
$$

The second term on the right hand side of Eq. (6) trivially separates in a term depending only on $Q$ and a term depending only on the relative variables, as the following equality holds true:

$$
\sum_{n=1}^{N} \sqrt{\lambda_{n}} q_{n} w_{n}(t)=Q \sum_{n=1}^{N} \sqrt{\lambda_{n}} w_{n}(t)+\sum_{n=1}^{N-1} \sqrt{\lambda_{n}} \tilde{q}_{n}\left(w_{n}(t)-\frac{\lambda_{n}}{\lambda_{N}} w_{N}(t)\right) .
$$

The above expression suggests to define the following new stochastic processes:

$$
\begin{aligned}
w(t) & =\frac{1}{\sqrt{\lambda}} \sum_{n=1}^{N} \sqrt{\lambda_{n}} w_{n}(t), \\
\tilde{w}_{n}(t) & =w_{n}(t)-\frac{\lambda_{n}}{\lambda_{N}} w_{N}(t), \quad n=1,2, \ldots, N-1,
\end{aligned}
$$

with $\lambda$ yet to be defined. The first noise is associated to the center-of-mass motion, the remaining ones are associated to the relative motion. In such a way, Eq. (31) becomes:

$$
\sum_{n=1}^{N} \sqrt{\lambda_{n}} q_{n} w_{n}(t)=\sqrt{\lambda} Q w(t)+\sum_{n=1}^{N-1} \sqrt{\lambda_{n}} \tilde{q}_{n} \tilde{w}_{n}(t) .
$$

The new noises here introduced are still Gaussian processes with zero mean; a crucial role is of course played by their second moments. The correlation function between $w(t)$ and $\tilde{w}_{n}(t)$ is equal to:

$$
\mathbb{E}_{\mathbb{Q}}\left[w(t) \tilde{w}_{n}(s)\right]=\frac{1}{\sqrt{\lambda}} \sum_{m=1}^{N}\left[\sqrt{\lambda_{m}} D_{m n}(t, s)-\sqrt{\frac{\lambda_{n} \lambda_{m}}{\lambda_{N}}} D_{m N}(t, s)\right], \quad n=1,2, \ldots N-1 .
$$


As we shall see soon, the necessary condition in order for the two types of motion to decouple is that the above expression vanishes

$$
\sum_{m=1}^{N}\left[\sqrt{\lambda_{m}} D_{m n}(t, s)-\sqrt{\frac{\lambda_{n} \lambda_{m}}{\lambda_{N}}} D_{m N}(t, s)\right]=0, \quad n=1,2, \ldots N-1,
$$

i.e. that $w(t)$ be statistically independent from the other noises. This condition is automatically satisfied if the original noises $w_{n}(t)$ are independent and identically distributed, i.e. if

$$
D_{n m}(t, s)=\delta_{n m} D(t, s),
$$

which is what it is usually assumed in white-noise collapse models (see Eq. (5)). Throughout the rest of the section we will assume that this condition is satisfied.

The correlation function of $w(t)$ is equal to:

$$
\mathbb{E}_{\mathbb{Q}}[w(t) w(s)]=\frac{1}{\lambda}\left(\sum_{n=1}^{N} \lambda_{n}\right) D(t, s) ;
$$

this expression suggests to set the value of the coupling constant $\lambda$ first introduced in Eq. (32) equal to:

$$
\lambda \equiv \sum_{n=1}^{N} \lambda_{n}=\frac{M}{m_{0}} \lambda_{0}
$$

so that the correlation function of the center-of-mass noise is identical to that of the noises associated to each single constituent. Thus, in accordance with what happens in the white noise case, the collapse coupling constant associated to the center-of-mass motion is proportional to the total mass of the system; as we shall see in the subsequent sections, this means that the collapse mechanism scales with the size of the system.

According to the previous assumptions, the correlation functions of the noises $\tilde{w}_{n}(t)$ are equal to:

$$
\begin{aligned}
\mathbb{E}_{\mathbb{Q}}\left[\tilde{w}_{n}(t) \tilde{w}_{m}(s)\right] & =C_{n m} D(t, s), \\
C_{n m} & \equiv \delta_{n m}+\frac{\sqrt{\lambda_{n} \lambda_{m}}}{\lambda_{N}},
\end{aligned}
$$

with $n, m=1,2, \ldots, N-1$. It easy to prove that the matrix $C$, having $C_{n m}$ as coefficients, is positive-definite. In fact one can write $C=I-\mathbf{X} \mathbf{Y}^{\top}$, where $\mathbf{X}, \mathbf{Y}$ are two $N-$ 1-dimensional vectors, $\mathbf{Y}=-\mathbf{X}$ and

$$
\mathbf{X}=\frac{1}{\sqrt{\lambda_{N}}}\left(\begin{array}{c}
\sqrt{\lambda_{1}} \\
\vdots \\
\sqrt{\lambda_{N-1}}
\end{array}\right)
$$


According to Statement 3.5.14 at page 93 in [27], $C$ is positive definite iff $\mathbf{X}^{\top} \mathbf{Y}<1$, inequality that can be easily verified with our definitions of $\mathbf{X}$ and $\mathbf{Y}$ :

$$
\mathbf{X}^{\top} \mathbf{Y}=-\frac{1}{\lambda_{N}} \sum_{i=1}^{N-1} \lambda_{i}=1-\frac{\lambda}{\lambda_{N}}<1 .
$$

Moreover, $C$ is real and symmetric, thus there exists an orthogonal matrix $O$ which diagonalizes it:

$$
O^{\top} C O=D, \quad D=\operatorname{diag}\left(d_{1}, \ldots d_{N-1}\right)
$$

where the eigenvalues $d_{i}$ of the matrix $D$ are real and positive, since $C$ is positive definite.

It is convenient to "diagonalize" the processes $\tilde{w}_{1}(t), \ldots, \tilde{w}_{N-1}(t)$ by defining the new stochastic processes:

$$
\bar{w}_{n}(t) \equiv \frac{1}{\sqrt{d_{n}}} \sum_{m=1}^{N-1} O_{n m}^{\top} \tilde{w}_{m}(t)
$$

an easy calculation shows that they are independent and identically distributed Gaussian processes with zero mean and correlation function equal to $D(t, s)$. By defining also:

$$
\bar{q}_{n} \equiv \frac{1}{\sqrt{\lambda_{n}}} \sum_{m=1}^{N-1} O_{n m}^{\top} \sqrt{\lambda_{m}} \tilde{q}_{m}, \quad \bar{\lambda}_{n}=\lambda_{n} d_{n}^{2},
$$

one can easily verify that the second term on the right hand side of Eq. (34) can be rewritten as:

$$
\sum_{n=1}^{N-1} \sqrt{\lambda_{n}} \tilde{q}_{n} \tilde{w}_{n}(t)=\sum_{n=1}^{N-1} \sqrt{\bar{\lambda}_{n}} \bar{q}_{n} \bar{w}_{n}(t)
$$

To summarize, the second term on the right hand side of Eq. (6) becomes

$$
\sum_{n=1}^{N} \sqrt{\lambda_{n}} q_{n} w_{n}(t)=\sqrt{\lambda} Q w(t)+\sum_{n=1}^{N-1} \sqrt{\bar{\lambda}_{n}} \bar{q}_{n} \bar{w}_{n}(t) .
$$

We now examine the third term of Eq. (6) . After some easy calculations, one finds the following result:

$$
\begin{aligned}
& \sum_{n, m=1}^{N} \sqrt{\lambda_{n}} q_{n} \int_{0}^{t} d s D_{n m}(t, s) \frac{\delta}{\delta w_{m}(s)}= \\
& =\sqrt{\lambda} Q \int_{0}^{t} d s \mathbb{E}_{\mathbb{Q}}[w(t) w(s)] \frac{\delta}{\delta w(s)}+\sqrt{\lambda} Q \sum_{n=1}^{N-1} \int_{0}^{t} d s \mathbb{E}_{\mathbb{Q}}\left[w(t) \tilde{w}_{n}(s)\right] \frac{\delta}{\delta \tilde{w}_{n}(s)} \\
& +\sum_{n=1}^{N-1} \sqrt{\lambda_{n}} \tilde{q}_{n} \int_{0}^{t} d s \mathbb{E}_{\mathbb{Q}}\left[\tilde{w}_{n}(t) w(s)\right] \frac{\delta}{\delta w(s)}+\sum_{n, m=1}^{N-1} \sqrt{\lambda_{n}} \tilde{q}_{n} \int_{0}^{t} d s \mathbb{E}_{\mathbb{Q}}\left[\tilde{w}_{n}(t) \tilde{w}_{m}(s)\right] \frac{\delta}{\delta \tilde{w}_{m}(s)} .
\end{aligned}
$$


As we see, the second and third term at the right hand side of the above equation vanish when condition (36) is satisfied, in which case the variables relative to the motion of the center of mass and those relative to the motion of the relative degrees of freedom decouple. This is the desired result. Moreover, under condition (37) one can rewrite Eq. (49) as follows:

$$
\begin{aligned}
& \sum_{n, m=1}^{N} \sqrt{\lambda_{n}} q_{n} \int_{0}^{t} d s D_{n m}(t, s) \frac{\delta}{\delta w_{m}(s)}= \\
& =\sqrt{\lambda} Q \int_{0}^{t} d s D(t, s) \frac{\delta}{\delta w(s)}+\sum_{n, m=1}^{N-1} \sqrt{\lambda_{n}} \tilde{q}_{n} \int_{0}^{t} d s C_{n m} D(t, s) \frac{\delta}{\delta \tilde{w}_{m}(s)} \\
& =\sqrt{\lambda} Q \int_{0}^{t} d s D(t, s) \frac{\delta}{\delta w(s)}+\sum_{n}^{N-1} \sqrt{\bar{\lambda}_{n}} \bar{q}_{n} \int_{0}^{t} d s D(t, s) \frac{\delta}{\delta \bar{w}_{n}(s)} .
\end{aligned}
$$

To summarize the results so far obtained, we have seen that when condition (36) is satisfied, the center of mass motion and the relative motion decouple; under the simplifying assumption (37), the equation for the center of mass becomes:

$$
\frac{d}{d t} \phi_{t}^{\mathrm{cm}}=\left[-\frac{i}{\hbar} H_{\mathrm{cm}}+\sqrt{\lambda} Q w(t)-2 \sqrt{\lambda} Q \int_{0}^{t} d s D(t, s) \frac{\delta}{\delta w(s)}\right] \phi_{t}^{\mathrm{cm}}
$$

where $w(t)$ and $\lambda$ have been defined in (32) and (39) respectively. The equation for the relative motion instead becomes:

$$
\frac{d}{d t} \phi_{t}^{\mathrm{rel}}=\left[-\frac{i}{\hbar} H_{\mathrm{rel}}+\sum_{n=1}^{N} \sqrt{\bar{\lambda}_{n}} \bar{q}_{n} \bar{w}_{n}(t)-2 \sum_{n, m=1}^{N-1} \sqrt{\bar{\lambda}_{n}} \bar{q}_{n} C_{n m} \int_{0}^{t} d s D(t, s) \frac{\delta}{\delta \bar{w}_{m}(s)}\right] \phi_{t}^{\mathrm{rel}},
$$

where $\bar{w}_{n}(t)$ and $\bar{\lambda}_{n}$ have been defined in (45) and (46) respectively.

We stress once more a crucial feature of the model, the amplification mechanism: according to Eq. (39), the coupling between the noise and the center of mass, thus the collapse strenght, scales with the size of the system. On the other side, relative degrees of freedom are coupled to the noise through $\lambda_{n}$, which do not scale with the size of the system, and remain small. This is the way in which the model described by Eq. (6) describes both classical properties of macroscopic objects (large values of $m$ ) and quantum properties of microscopic systems (small values of $m$ ).

\section{SOLUTION OF THE EQUATION FOR A FREE QUANTUM PARTICLE}

Now we restrict our attention to the dynamics of a free quantum particle of mass $m$. Eq. (6) then becomes:

$$
\frac{d}{d t} \phi_{t}(x)=\left[-\frac{i}{\hbar} \frac{p^{2}}{2 m}+\sqrt{\lambda} q w(t)-2 \sqrt{\lambda} q \int_{0}^{t} d s D(t, s) \frac{\delta}{\delta w(s)}\right] \phi_{t}(x),
$$


where $D(t, s)$ is the correlation function of the Gaussian noise $w(t)$. As discussed in the previous section, this same equation describes also the dynamics of the center of mass of an isolated system (in particular, a macroscopic object) of total mass $m$.

Aim of this section is to find the explicit solution of Eq. (53). In [26] it has been shown that the Green's function $G\left(x, t ; x_{0}, 0\right)$ of Eq. (53) allows for the following path-integral representation:

$$
G\left(x, t ; x_{0}, 0\right)=\int_{q(0)=x_{0}}^{q(t)=x} \mathcal{D}[q] e^{\mathcal{S}[q]},
$$

where, as indicated, the integration is carried out over all the paths connecting $q(0)=x_{0}$ to $q(t)=x$. The 'action' $\mathcal{S}[q]$, which contrary to the standard quantum case, has both a real and an imaginary part, is:

$$
\mathcal{S}[q]=\int_{0}^{t} d s\left[\frac{i m}{2 \hbar} q^{\prime 2}(s)+\sqrt{\lambda} q(s) w(s)-\lambda q(s) \int_{0}^{t} d r q(r) D(s, r)\right] .
$$

That Eq. (55) represents the correct action associated to Eq. (53) can be easily verified by checking that the wave function

$$
\phi_{t}(x)=\int_{-\infty}^{\infty} d x_{0} G\left(x, t ; x_{0}, 0\right) \phi_{0}\left(x_{0}\right)
$$

solves Eq. (53) . The advantage of the path integral representation of the Green's function, with respect to the standard representation associated to a differential equation like (53), is that it avoids resorting to the functional derivative of the noise, which is a source of major complications. We now compute the path integration in (54).

Following the standard Feynman polygonal approach [28], we divide the time interval $[0, t]$ in $N$ subintervals, each of length $\epsilon=t / N$; the intermediate time points are defined as: $t_{k}=k \epsilon$. The path integral is then understood as the limit $N \rightarrow \infty$ of a multiple integral over the $N-1$ variables $q_{k}=q\left(t_{k}\right), k=1, \ldots, N-1:$

$$
G\left(x, t ; x_{0}, 0\right)=\lim _{N \rightarrow \infty} G_{N}\left(x, t ; x_{0}, 0\right)
$$

with

$$
G_{N}\left(x, t ; x_{0}, 0\right)=\left(\frac{m}{2 \pi i \hbar \epsilon}\right)^{\frac{N}{2}} \int \cdots \int \prod_{k=1}^{N-1} d q_{k} e^{\mathcal{S}_{N}[q]},
$$

and $\mathcal{S}_{N}[q]$ is the discretized form of the 'action', which reads:

$$
\mathcal{S}_{N}[q]=\sum_{k=1}^{N}\left[\frac{i}{\hbar} \frac{m}{2 \epsilon}\left(q_{k}-q_{k-1}\right)^{2}+\epsilon \sqrt{\lambda} w_{k} q_{k}-\epsilon^{2} \lambda q_{k} \sum_{j=1}^{N} D_{k, j} q_{j}\right]
$$


where $w_{k}=w\left(t_{k}\right)$ and $D_{k, j}=D\left(t_{k}, t_{j}\right)$. The constraints are: $q_{0}=x_{0}$ and $q_{N}=x$. We re-write $G_{N}\left(x, t ; x_{0}, 0\right)$, separating the terms which are constant with respect to the integration variables, the linear terms and the quadratic terms; using a vector notation, we have:

$$
\begin{aligned}
& G_{N}\left(x, t ; x_{0}, 0\right)=\left(\frac{m}{2 \pi i \hbar \epsilon}\right)^{\frac{N}{2}} \exp \left[\frac{i m}{2 \hbar \epsilon}\left(x_{0}^{2}+x^{2}\right)\right.\left.+\epsilon \sqrt{\lambda} w_{N} x-\epsilon^{2} \lambda x^{2} D_{N, N}\right] \\
& \cdot \int_{-\infty}^{+\infty} d \mathbf{X} \exp [-\mathbf{X} \cdot A \mathbf{X}+2 \mathbf{X} \cdot \mathbf{Y}],
\end{aligned}
$$

where $\mathbf{X}$ and $\mathbf{Y}$ are two $(N-1)$-dimensional vectors defined as follows:

$$
\mathbf{X}=\left(\begin{array}{c}
q_{1} \\
q_{2} \\
\vdots \\
q_{N-2} \\
q_{N-1}
\end{array}\right), \quad \mathbf{Y}=-\frac{i m}{2 \hbar \epsilon}\left(\begin{array}{c}
x_{0} \\
0 \\
\vdots \\
0 \\
x
\end{array}\right)+\frac{\epsilon \sqrt{\lambda}}{2}\left(\begin{array}{c}
w_{1} \\
w_{2} \\
\vdots \\
w_{N-2} \\
w_{N-1}
\end{array}\right)-\frac{\epsilon^{2} \lambda}{2} x\left(\begin{array}{c}
D_{1, N} \\
D_{2, N} \\
\vdots \\
D_{N-2, N} \\
D_{N-1, N}
\end{array}\right) .
$$

We have also used the short hand notation: $d \mathbf{X}=\prod_{k=1}^{N-1} d q_{k}$. The matrix $A$ is the sum of two symmetric, $(N-1)$-dimensional square matrices $B$ and $C$, whose entries are:

$$
\begin{aligned}
B_{i, i} & =-\frac{i m}{\hbar \epsilon}, \quad B_{i, i \pm 1}=\frac{i m}{2 \hbar \epsilon}, \\
B_{i, j} & =0, \quad j \neq i, i \pm 1, \\
C_{i, j} & =\epsilon^{2} \lambda D_{i, j} .
\end{aligned}
$$

The multiple Gaussian integral of Eq. (60) can be immediately evaluated by using the standard result:

$$
\int_{-\infty}^{+\infty} d \mathbf{X} \exp [-\mathbf{X} \cdot A \mathbf{X}+a \mathbf{X} \cdot \mathbf{Y}]=\sqrt{\frac{\pi^{N-1}}{\operatorname{det}(A)}} \exp \left[\frac{a^{2}}{4} \mathbf{Y} \cdot A^{-1} \mathbf{Y}\right]
$$

$G_{N}\left(x, t ; x_{0}, 0\right)$ then becomes:

$$
G_{N}\left(x, t ; x_{0}, 0\right)=\sqrt{\frac{(m / 2 i \hbar \epsilon)^{N}}{\pi \operatorname{det}(A)}} \exp \left[\mathbf{Y} \cdot A^{-1} \mathbf{Y}+\frac{i m}{2 \hbar \epsilon}\left(x_{0}^{2}+x^{2}\right)+\epsilon \sqrt{\lambda} w_{N} x-\epsilon^{2} \lambda x^{2} D_{N, N}\right] .
$$

Note that the integral in Eq. (65) exists only if $A$ is a positive definite matrix. The result can be extended to non-negative matrices, following e.g. the procedure of Theorem 1, page 13 of [19]. Accordingly, our results strictly hold only when the correlation function $D(t, s)$ of the noise $w(t)$, seen as an integral kernel, is non-negative definite.

The next step is to take the limit $N \rightarrow \infty$, in which case one encounters two main difficulties: the first is to evaluate the inverse matrix $A^{-1}$, the second is to compute the determinant of $A$, in both cases for any $N$. To solve these difficulties, we proceed as in [29]. 
In order to evaluate $\mathbf{Y} \cdot A^{-1} \mathbf{Y}$, we introduce a twice differentiable function $z(s), s \in[0, t]$, yet to be determined, such that, given the vector $\mathbf{Z}:=\left(z_{1}, \ldots z_{N-1}\right)^{\top}$, with $z_{k}=z\left(t_{k}\right), k=1, \ldots N-1$, the following matrix equation is satisfied:

$$
A \mathbf{Z}=\mathbf{Y}
$$

For reasons which will be clear soon, we will set $z(0)=x_{0}$ and $z(t)=x$. We then have:

$$
\begin{aligned}
\mathbf{Y} \cdot A^{-1} \mathbf{Y}= & \mathbf{Y} \cdot \mathbf{Z} \\
= & -\frac{i m}{2 \hbar \epsilon}\left(x_{0} z_{1}+x z_{N-1}\right)+\frac{\epsilon \sqrt{\lambda}}{2} \sum_{j=1}^{N-1} w_{j} z_{j}-\frac{\epsilon^{2} \lambda}{2} x \sum_{j=1}^{N-1} D_{j, N} z_{j} \\
= & -\frac{i m}{2 \hbar \epsilon} x_{0}\left(z(0)+\epsilon z^{\prime}(0)+O\left(\epsilon^{2}\right)\right)-\frac{i m}{2 \hbar \epsilon} x\left(z(t)-\epsilon z^{\prime}(t)+O\left(\epsilon^{2}\right)\right)+ \\
& +\frac{\epsilon \sqrt{\lambda}}{2} \sum_{j=1}^{N-1} w_{j} z_{j}-\frac{\epsilon^{2} \lambda}{2} x \sum_{j=1}^{N-1} D_{j, N} z_{j}
\end{aligned}
$$

the prime in $z^{\prime}(s)$ denotes the first derivative of $z(s)$. Inserting the above result in (66), we have:

$$
G_{N}\left(x, t ; x_{0}, 0\right)=\sqrt{\frac{(m / 2 i \hbar \epsilon)^{N}}{\pi \operatorname{det}(A)}} \exp \left[-\frac{i m}{2 \hbar}\left(x_{0} z^{\prime}(0)-x z^{\prime}(t)\right)+\frac{\epsilon \sqrt{\lambda}}{2} \sum_{j=1}^{N-1} w_{j} z_{j}+O(\epsilon)\right],
$$

where we have collected all terms of order $\epsilon$ or higher, which will vanish in the limit $N \rightarrow \infty$. Note that the two terms of (66) proportional to $x_{0}^{2}$ and $x^{2}$, which are of order $\epsilon^{-1}$, are canceled by the analogous terms in (68): this is a consequence of the boundary conditions we have set on the function $z(s)$.

In order to compute the determinant of the matrix $A=B+C$, where $B$ and $C$ have been defined in (62) and (64), it is convenient to isolate the determinant of $B$ by writing $A=B\left(I+B^{-1} C\right)$, so that:

$$
\operatorname{det}(A)=\operatorname{det}(B) \operatorname{det}\left(I+B^{-1} C\right)
$$

The quantity $\operatorname{det}(B)$ will be used to simplify part of the terms under the square root in (69). The matrix $B$ is equal to $B=\frac{m}{2 i \hbar \epsilon} \bar{B}$ with

$$
\bar{B}_{i, i}=2, \quad \bar{B}_{i, i \pm 1}=-1, \quad \bar{B}_{i, j}=0, \quad j \neq i, i \pm 1 .
$$

Let us call $\Delta_{k}$ the determinant of the matrix obtained from $\bar{B}$ by removing the first $N-k-1$ rows and columns; the following recursive relation is easy to prove:

$$
\Delta_{k}=2 \Delta_{k-1}-\Delta_{k-2}, \quad k=1, \ldots N-1,
$$


where we have set $\Delta_{0}=1$ and $\Delta_{-1}=0$. From this relation, by induction one immediately sees that $\Delta_{k}=k+1$, which means that $\operatorname{det}(\bar{B})=N$. Accordingly we have:

$$
\operatorname{det}(B)=N\left(\frac{m}{2 i \hbar \epsilon}\right)^{N-1}
$$

Collecting all results we can write:

$$
G_{N}\left(x, t ; x_{0}, 0\right)=\sqrt{\frac{m}{2 i \pi \hbar t u_{N}(t)}} \exp \left[-\frac{i m}{2 \hbar}\left(x_{0} z^{\prime}(0)-x z^{\prime}(t)\right)+\frac{\epsilon \sqrt{\lambda}}{2} \sum_{j=1}^{N-1} w_{j} z_{j}+O(\epsilon)\right]
$$

where $t=N \epsilon$, and we have introduced the quantity $u_{N}(t):=\operatorname{det}\left(I+B^{-1} C\right)$. We are now in the position to perform the limit $N \rightarrow \infty$; the Green's function becomes:

$$
G\left(x, t ; x_{0}, 0\right)=\sqrt{\frac{m}{2 i \pi \hbar t u(t)}} \exp \left[-\frac{i m}{2 \hbar}\left(x_{0} z^{\prime}(0)-x z^{\prime}(t)\right)+\frac{\sqrt{\lambda}}{2} \int_{0}^{t} d s w(s) z(s)\right],
$$

which is defined in terms of the two unknown functions $z(s)$ and $u(t):=\lim _{N \rightarrow \infty} u_{N}(t)$. We now show how to determine them.

Written in components and once divided by $\epsilon$, Eq. (67) becomes:

$$
\frac{i m}{2 \hbar \epsilon^{2}}\left(z_{k+1}-2 z_{k}+z_{k-1}\right)+\epsilon \lambda \sum_{j=1}^{N-1} D_{k, j} z_{j}=\frac{\sqrt{\lambda}}{2} w_{k}-\frac{\epsilon \lambda}{2} x D_{k, N}, \quad k=1, \ldots N-1 ;
$$

note that for $k=1$ and for $k=N-1$, consistency is assured thanks to the assumption $z_{0}=x_{0}$ and $z_{N}=x$. Taking the limit $\epsilon \rightarrow 0(N \rightarrow \infty)$ we obtain:

$$
\frac{i m}{2 \hbar} z^{\prime \prime}(s)+\lambda \int_{0}^{t} d r D(s, r) z(r)=\frac{\sqrt{\lambda}}{2} w(s)
$$

which is an integro-differential equation to be solved together with the conditions $z(0)=x_{0}$, $z(t)=x$. The above equation determines $z(s)$. In appendix $\mathrm{A}$ we prove that Eq. (77) admits a unique solution.

Let us now determine $u(t)$; we follow once again the procedure outlined in [29]. According to Statement 11.11.4, page 451 of [27] one can write

$$
\operatorname{det}(I-\eta K)=\exp \left[\int_{\eta}^{0} \operatorname{Tr}[R(\mu)] d \mu\right]
$$

where $R(\mu)$ is a matrix satisfying the equation $R(\mu)=K+\mu K R$. Applied to our problem, we have:

$$
u_{N}(t)=\operatorname{det}\left(I+B^{-1} C\right)=\exp \left[\int_{-1}^{0}\left(\epsilon \sum_{k=1}^{N-1} R_{k, k}(\mu)\right) d \mu\right]
$$


in our case $K=B^{-1} C$, thus $R(\mu)$ satisfies the matrix equation $B R(\mu)=C \epsilon^{-1}+\mu C R(\mu)$. Written in components, and divided by $\epsilon$, this equation becomes:

$$
\frac{i m}{2 \hbar \epsilon^{2}}\left[R_{i-1, j}(\mu)-2 R_{i, j}(\mu)+R_{i+1, j}(\mu)\right]=\lambda D_{i, j}+\mu \epsilon \sum_{k=1}^{N-1} D_{i, k} R_{k, j}(\mu),
$$

with the boundary conditions $R_{0, j}(\mu)=R_{N, j}(\mu)=0$ for any $j$ and $\mu$. Taking the limit $\epsilon \rightarrow 0$, the matrix $R_{i, j}(\mu)$ becomes a function $R(s, r, \mu)$ satisfying the following integro-differential equation:

$$
\frac{i m}{2 \hbar} \frac{\partial^{2}}{\partial s^{2}} R(s, r, \mu)-\lambda \mu \int_{0}^{t} d l D(s, l) R(l, r, \mu)=\lambda D(s, r),
$$

which has to be solved together with the boundary conditions $R(0, r, \mu)=R(t, r, \mu)=0$ for any $r \in[0, t]$ and $\mu \in[-1,0]$. The existence and uniqueness theorem of appendix $\AA$ applies also to this equation. In the limit $N \rightarrow \infty$, Eq. (79) becomes:

$$
u(t)=\exp \left[\int_{-1}^{0} d \mu\left(\int_{0}^{t} d s R(s, s, \mu)\right)\right] .
$$

Eq. (75), with $z(s)$ defined by (77) and $u(t)$ defined by (82), gives a complete description of the Green's function of Eq. (53). This is the main result of this section.

The function $z(s)$ solution of Eq. (177) depends on the end points $x_{0}$ and $x$ because of the boundary conditions. This is not evident in the expression (75) for the propagator. In order to make such a dependence on $x_{0}$ and $x$ explicit, we rewrite $z(s)$ as follows:

$$
z(s)=f(s) x_{0}+g(s) x+h(s)
$$

where $f(s)$ satisfies the homogenous integro-differential equation:

$$
\frac{i m}{2 \hbar} f^{\prime \prime}(s)+\lambda \int_{0}^{t} d r D(s, r) f(r)=0
$$

with boundary conditions $f(0)=1, f(t)=0$, while $g(s)$ solves the same equation but with boundary conditions $g(0)=0, g(t)=1$. The function $h(s)$ instead satisfies the non-homogenous equation:

$$
\frac{i m}{2 \hbar} h^{\prime \prime}(s)+\lambda \int_{0}^{t} d r D(s, r) h(r)=\frac{\sqrt{\lambda}}{2} w(s)
$$

with boundary conditions $h(0)=h(t)=0$. Note that $f(s), g(s)$ and $h(s)$ depend also in the parameter $t$, so one should more properly write $f_{t}(s), g_{t}(s), h_{t}(s)$; sometimes we will omitt the pedex $t$ when no confusion arises. Here and in the following, the prime denotes differentiation with respect to the variable in parentheses. 
With the help of (83) one can write the Green's function of Eq. (75) in the following way:

$$
G\left(x, t ; x_{0}, 0\right)=\sqrt{\frac{m}{2 i \pi \hbar t u(t)}} \exp \left[-\mathcal{A}_{t} x_{0}^{2}-\tilde{\mathcal{A}}_{t} x^{2}+\mathcal{B}_{t} x_{0} x+\mathcal{C}_{t} x_{0}+\mathcal{D}_{t} x+\mathcal{E}_{t}\right],
$$

where the dependence on $x_{0}$ and $x$ has been made explicit. The coefficients $\mathcal{A}_{t}, \tilde{\mathcal{A}}_{t}$ and $\mathcal{B}_{t}$ are deterministic functions of time, defined as follows:

$$
\mathcal{A}_{t}=k f_{t}^{\prime}(0), \quad \tilde{\mathcal{A}}_{t}=-k g_{t}^{\prime}(t), \quad \mathcal{B}_{t}=k\left(f_{t}^{\prime}(t)-g_{t}^{\prime}(0)\right), \quad k=\frac{i m}{2 \hbar},
$$

while the coefficients $\mathcal{C}_{t}, \mathcal{D}_{t}$ and $\mathcal{E}_{t}$ are random processes:

$$
\begin{aligned}
\mathcal{C}_{t} & =-k h_{t}^{\prime}(0)+\frac{\sqrt{\lambda}}{2} \int_{0}^{t} w(l) f_{t}(l) d l \\
\mathcal{D}_{t} & =k h_{t}^{\prime}(t)+\frac{\sqrt{\lambda}}{2} \int_{0}^{t} w(l) g_{t}(l) d l \\
\mathcal{E}_{t} & =\frac{\sqrt{\lambda}}{2} \int_{0}^{t} w(l) h_{t}(l) d l .
\end{aligned}
$$

The propagator (86) takes a simpler and more symmetric form when the noise is time translation invariant: $D(t, s)=D(t-s)$; according to the symmetry in the $(t, s)$ variables one also has: $D(t-s)=D(|t-s|)$. In this case, one can easily verify that if $f(s)$ is solution of Eq. (84) with the boundary conditions $f(0)=1, f(t)=0$, then $\tilde{f}(s)=f(t-s)$ is still a solution Eq. (84), with boundary conditions $\tilde{f}(0)=0$ and $\tilde{f}(t)=1$; in other words: $\tilde{f}(s)=g(s)$. Then (83) becomes:

$$
z(s)=f(s) x_{0}+f(t-s) x-h(s)
$$

and the Green's function (86) simplifies as follows:

$$
G\left(x, t ; x_{0}, 0\right)=\sqrt{\frac{m}{2 i \pi \hbar t u(t)}} \exp \left[-\mathcal{A}_{t}\left(x_{0}^{2}+x^{2}\right)+\mathcal{B}_{t} x_{0} x+\mathcal{C}_{t} x_{0}+\mathcal{D}_{t} x+\mathcal{E}_{t}\right],
$$

where the deterministic coefficients now are:

$$
\mathcal{A}_{t}=k f_{t}^{\prime}(0), \quad \mathcal{B}_{t}=2 k f_{t}^{\prime}(t)
$$

and the stochastic terms read:

$$
\begin{aligned}
\mathcal{C}_{t} & =-k h_{t}^{\prime}(0)+\frac{\sqrt{\lambda}}{2} \int_{0}^{t} d l w(l) f_{t}(l), \\
\mathcal{D}_{t} & =k h_{t}^{\prime}(t)+\frac{\sqrt{\lambda}}{2} \int_{0}^{t} d l w(l) f_{t}(t-l), \\
\mathcal{E}_{t} & =\frac{\sqrt{\lambda}}{2} \int_{0}^{t} d l w(l) h_{t}(l) .
\end{aligned}
$$


Accordingly, for a general time translation invariant noise, the structure of the Green's function is the same as in the familiar white-noise case [10, 30], as we will review in Sec. V]. Note that due to linearity, the general solution of Eq. (85) can be written as $h(s)=h^{\mathrm{P}}(s)+h^{0}(s)$, where $h^{0}(s)$ solves the homogeneous equation (84) and $h^{\mathrm{P}}(s)$ is a particular solution of Eq. (85). Often the particular solution admits an integral expression of the form:

$$
h^{\mathrm{P}}(s)=\int_{0}^{s} d l r(s, l)
$$

where $r(s, l)$ is some function. Eq. (97) implies that $h^{\mathrm{P}}(0)=0$. One can immediately verify that, by taking $h^{0}(s)=-h^{\mathrm{P}}(t) f(t-s)$, the function:

$$
h(s)=h^{\mathrm{P}}(s)-h^{\mathrm{P}}(t) f(t-s),
$$

solves Eq. (85), with the correct boundary conditions. Thus also $h(s)$ can be expressed in terms of $f(s)$, once $h^{\mathrm{P}}(s)$ is given as in (97).

A concluding remark is at order. In this paper we have computed the Green's function $G\left(x, t ; x_{0}, 0\right)$ by exploiting the path-integral formalism. The same result can be obtained also by resorting to the standard operator formalism, starting with the expression for the propagator first proposed in [24, 25].

\section{THE NON MARKOVIAN STOCHASTIC SCHRÖDINGER EQUATION}

The result of the previous section allows us to rewrite Eq. (53) in a simpler form, removing the functional derivative which is not easy to handle. In this way, we will prove the correctness of the Ansatz introduced in Sec. 4.3 of Ref. [17], according to which the functional derivative can be replaced with a suitable function of the position and momentum operators.

The starting point is Eq. (56) where the solution $\phi_{t}(x)$ of Eq. (53) is expressed in terms of the Green's function $G\left(x, t ; x_{0}, 0\right)$. Using standard path-integral techniques, e.g. generalizing the procedure described at page 509 of [31], one can show that $G\left(x, t ; x_{0}, 0\right)$ given by (54), with $\mathcal{S}[q]$ given by (55), satisfies the following equation:

$$
\frac{d}{d t} G\left(x, t ; x_{0}, 0\right)=\left[-\frac{i}{\hbar} \frac{p^{2}}{2 m}+\sqrt{\lambda} x w(t)\right] G\left(x, t ; x_{0}, 0\right)+\tilde{G}\left(x, t ; x_{0}, 0\right),
$$

with:

$$
\tilde{G}\left(x, t ; x_{0}, 0\right):=-2 \lambda x \int_{q(0)=x_{0}}^{q(t)=x} \mathcal{D}[q] \int_{0}^{t} d s q(s) D(t, s) e^{\mathcal{S}[q]} .
$$


The path integral in (100) cannot be trivially reduced to (54) because $q(s)$ appears, which depends on the entire time interval $[0, t]$. Thus we have to re-calculate $\tilde{G}\left(x, t ; x_{0}, 0\right)$ as we did with $G\left(x, t ; x_{0}, 0\right)$. Proceeding as in Section III, we define $\tilde{G}\left(x, t ; x_{0}, 0\right)=\lim _{N \rightarrow \infty} \tilde{G}_{N}\left(x, t ; x_{0}, 0\right)$, where $\tilde{G}_{N}\left(x, t ; x_{0}, 0\right)$ is:

$$
\begin{aligned}
\tilde{G}_{N}\left(x, t ; x_{0}, 0\right)= & -2 \lambda q\left(\frac{m}{2 \pi i \hbar \epsilon}\right)^{\frac{N}{2}} \exp \left[\frac{i m}{2 \hbar \epsilon}\left(x_{0}^{2}+x^{2}\right)+\epsilon \sqrt{\lambda} w_{N} x-\epsilon^{2} \lambda x^{2} D_{N, N}\right] \\
& \int_{-\infty}^{+\infty} d \mathbf{X}\left(\epsilon \sum_{k=1}^{N-1} \mathbf{K}^{\top} \mathbf{X}+O(\epsilon)\right) \exp [-\mathbf{X} \cdot A \mathbf{X}+2 \mathbf{X} \cdot \mathbf{Y}]
\end{aligned}
$$

where $O(\epsilon)$ collects all terms of order $\epsilon, \mathbf{K}^{\top}=\left(0, \ldots D_{N k}, \ldots 0\right)$ where $D_{N k}$ is placed in the $k$-th position, and $\mathbf{X}, \mathbf{Y}, A$ are defined in Eqs. (61) and (62)-(64). Applying the following generalization of Eq. (65):

$$
\int_{-\infty}^{+\infty} d \mathbf{X} \mathbf{K}^{\top} \mathbf{X} \exp [-\mathbf{X} \cdot A \mathbf{X}+a \mathbf{X} \cdot \mathbf{Y}]=\sqrt{\frac{\pi^{N-1}}{\operatorname{det}(A)}} \frac{a}{2} \mathbf{K}^{\top} A^{-1} \mathbf{Y} \exp \left[\frac{a^{2}}{4} \mathbf{Y} \cdot A^{-1} \mathbf{Y}\right]
$$

and taking the limit for $\epsilon \rightarrow 0$ one finds that

$$
\tilde{G}\left(x, t ; x_{0}, 0\right)=-2 \lambda x \int_{0}^{t} d s z(s) D(t, s) G\left(x, t ; x_{0}, 0\right),
$$

where $z(s)$ is defined in Eq. (177). Thus, using (99) and (103) one can formally write $(x=q$, the position operator):

$$
\frac{d}{d t} G\left(x, t ; x_{0}, 0\right)=\left[-\frac{i}{\hbar} \frac{p^{2}}{2 m}+\sqrt{\lambda} q w(t)-2 \lambda q \int_{0}^{t} d s z(s) D(t, s)\right] G\left(x, t ; x_{0}, 0\right) .
$$

Note that it is possible to find this same result by inserting $G\left(x, t ; x_{0}, 0\right)$ directly into Eq. (53). However, to reach the result of Eq. (104), one encounters a term of the form $\delta z^{\prime}(l) / \delta w(s)$ which can be simplified only under the assumption that the time derivative and the functional derivative acting on $z(s)$ commute. This is not true in general, and one has to check it by finding the explicit functional dependence of $z(s)$ on $w(s)$. This fact makes this second approach more cumbersome than the one we have followed.

Eq. (104) is not written in a useful way yet, since $z(s)$ depends both on $x_{0}$ and $x$. We make explicit this dependence on $x_{0}$ and $x=q$ by resorting to (83):

$$
\tilde{G}\left(x, t ; x_{0}, 0\right)=-2 \lambda x\left[\int_{0}^{t} d s D(t, s)\left(f(s) x_{0}+g(s) x+h(s)\right)\right] G\left(x, t ; x_{0}, 0\right) .
$$

The term in (105) depending on $x_{0}$ does not allow to perform the integration over the $x_{0}$ variable, to compute $\phi_{t}$ from (56). This term can be quite easily written in terms of the position operator 
$q$ and the momentum operator $p=-i \hbar \frac{\partial}{\partial x}$ acting on $G\left(x, t ; x_{0}, 0\right)$, as follows:

$$
\begin{aligned}
x_{0} G\left(x, t ; x_{0}, 0\right)=\frac{1}{g^{\prime}(0)-f^{\prime}(t)}\left[\frac{2 i \hbar}{m} \frac{\partial}{\partial x} G\left(x, t ; x_{0}, 0\right)\right. \\
\left.\quad+\left(2 x g^{\prime}(t)+h^{\prime}(t)+\frac{i \sqrt{\lambda} \hbar}{m} \int_{0}^{t} d l w(l) g(l)\right) G\left(x, t ; x_{0}, 0\right)\right] .
\end{aligned}
$$

Collecting all the above results, we then find that $\phi_{t}(x)$ satisfies the following equation:

$$
\frac{d}{d t} \phi_{t}(x)=\left[-\frac{i}{\hbar} \frac{p^{2}}{2 m}+\sqrt{\lambda} q w(t)-2 \lambda q \int_{0}^{t} d s D(t, s)\left(q a_{t}(s)+p b_{t}(s)+c_{t}(s)\right)\right] \phi_{t}(x),
$$

where

$$
\begin{aligned}
& a_{t}(s)=g(s)+2 \frac{g^{\prime}(t)}{g^{\prime}(0)-f^{\prime}(t)} f(s), \\
& b_{t}(s)=-\frac{2}{m} \frac{f(s)}{g^{\prime}(0)-f^{\prime}(t)}, \\
& c_{t}(s)=h(s)+\frac{f(s)}{g^{\prime}(0)-f^{\prime}(t)}\left(h^{\prime}(t)+\frac{i \sqrt{\lambda} \hbar}{m} \int_{0}^{t} d l w(l) g(l)\right) .
\end{aligned}
$$

This is the non-Markovian stochastic Schrödinger equation, whose Green's function is given by Eq. (86). When the noise is time-translation invariant $(D(t, s)=D(|t-s|))$, the functions $a_{t}(s)$, $b_{t}(s), c_{t}(s)$ simplify as follows:

$$
\begin{aligned}
& a_{t}(s)=f(t-s)+\frac{f^{\prime}(0)}{f^{\prime}(t)} f(s), \\
& b_{t}(s)=\frac{1}{m} \frac{f(s)}{f^{\prime}(t)} \\
& c_{t}(s)=h(s)-\frac{f(s)}{2 f^{\prime}(t)}\left(h^{\prime}(t)+\frac{i \sqrt{\lambda} \hbar}{m} \int_{0}^{t} d l w(l) f(t-l)\right) .
\end{aligned}
$$

As anticipated, Eq. (106) agrees with the ansatz first proposed in [17].

\section{WHITE NOISE LIMIT OF THE GREEN'S FUNCTION}

Having found the Green's function associated to Eq. (53), the first task is to check that, in the white noise limit, it coincides with the propagator already known in the literature [10, 30]. Since $D(s, r)=\delta(s-r)$ is time translation invariant, we can use the expression (91) for $z(s)$. In the white noise case, Eq. (84) reduces to the second order differential equation:

$$
f^{\prime \prime}(s)-\frac{2 i \lambda \hbar}{m} f(s)=0, \quad f(0)=1, \quad f(t)=0,
$$


whose solution can be easily computed:

$$
\begin{aligned}
f(s) & =\cosh v s-\operatorname{coth} v t \sinh v s \\
v & :=\frac{1+i}{2} \omega, \quad \omega:=2 \sqrt{\frac{\hbar \lambda}{m}} .
\end{aligned}
$$

Eq. (85) for $h(s)$ instead reduces to:

$$
h^{\prime \prime}(s)-\frac{2 i \lambda \hbar}{m} h(s)=-\frac{i \hbar}{m} \sqrt{\lambda} w(s), \quad h(0)=h(t)=0 .
$$

Using once again time translation invariance, we know that $h(s)$ takes the form (98), where a particular solution $h^{\mathrm{P}}(s)$ is [32]:

$$
h^{\mathrm{P}}(s)=-\frac{i \hbar}{m v} \sqrt{\lambda} \int_{0}^{s} d r w(r) \sinh v(s-r) .
$$

The Green's function then has the form (92), where the deterministic coefficients $\mathcal{A}_{t}$ and $\mathcal{B}_{t}$ are equal to:

$$
\mathcal{A}_{t}=\frac{\lambda}{v} \operatorname{coth} v t, \quad \mathcal{B}_{t}=\frac{2 \lambda}{v} \sinh ^{-1} v t
$$

while the random coefficients $\mathcal{C}_{t}, \mathcal{D}_{t}$ and $\mathcal{E}_{t}$ are equal to:

$$
\begin{aligned}
\mathcal{C}_{t}= & \sqrt{\lambda} \int_{0}^{t} d l w(l) \frac{\sinh v(t-l)}{\sinh v t}, \\
\mathcal{D}_{t}= & \sqrt{\lambda} \int_{0}^{t} d l w(l) \frac{\sinh v l}{\sinh v t}, \\
\mathcal{E}_{t}= & \frac{v}{4}\left(\int_{0}^{t} d l w(l) \sinh v l \int_{0}^{t} d s w(s) \frac{\sinh v(t-s)}{\sinh v t}\right. \\
& \left.-\int_{0}^{t} d l w(l) \int_{0}^{l} d s w(s) \sinh v(l-s)\right) .
\end{aligned}
$$

After some manipulation and an integration by parts, one can rewrite $\mathcal{E}_{t}$ as follows:

$$
\begin{aligned}
\mathcal{E}_{t} & =\frac{v}{2} \int_{0}^{t} d l w(l) \frac{\sinh v(t-l)}{\sinh v t} \int_{0}^{l} d s w(s) \sinh v s \\
& =\frac{v^{2}}{4} \int_{0}^{t} d l \frac{\left(\int_{0}^{l} d s w(s) \sinh v s\right)^{2}}{\sinh ^{2} v l} \\
& =\frac{v^{2}}{4 \lambda} \int_{0}^{t} d l \mathcal{D}_{l} .
\end{aligned}
$$

When $\mathcal{E}_{t}$ is written as in Eq. (122), then the Green's function (75) reduces to that of Eq. 6.2.42, page 180 of [30]. When $\mathcal{E}_{t}$ is rewritten as in Eq. (123), it coincides with the corresponding term of [10]. 
We now compute $R(s, r, \mu)$, which in turn determines $u(t)$. In the white noise limit, Eq. (81) becomes:

$$
\frac{\partial^{2}}{\partial s^{2}} R(s, r, \mu)+v^{2} \mu R(s, r, \mu)=-v^{2} \delta(s-r),
$$

with $R(0, r, \mu)=R(t, r, \mu)=0$ for any $r, \mu . R(s, r, \mu)$ clearly displays a discontinuity on its first derivative for $s=r$. The solution is found by imposing on the solution of the homogeneous equation the correct discontinuity conditions on the first derivative, together with the boundary conditions. $R(s, r, \mu)$, having the correct boundary conditions in $s=0$ and $s=t$, is easily determined:

$$
R(s, r, \mu)= \begin{cases}a \sinh i v \sqrt{\mu} s \quad s \leq r, & \\ b \sinh i v \sqrt{\mu}(s-t) & s \geq r,\end{cases}
$$

where $a$ and $b$ are two constants determined by the following relations:

$$
\left\{\begin{array}{l}
a \sinh i v \sqrt{\mu} r=b \sinh i v \sqrt{\mu}(r-t), \\
b \sqrt{\mu} \cosh i v \sqrt{\mu}(r-t)-a \sqrt{\mu} \cosh i v \sqrt{\mu} r=-\frac{2 i}{m v},
\end{array}\right.
$$

the first equation represents the continuity of $R(s, r, \mu)$ in $r$ and the second equation encodes the discontinuity of the first derivative.

After some algebra on finds the following result:

$$
R(s, r, \mu)= \begin{cases}\frac{i v}{\sqrt{\mu}} \frac{\sinh i v \sqrt{\mu}(r-t) \sinh i v \sqrt{\mu} s}{\sinh i v \sqrt{\mu} t} & s \leq r, \\ \frac{i v}{\sqrt{\mu}} \frac{\sinh i v \sqrt{\mu} r \sinh i v \sqrt{\mu}(s-t)}{\sinh i v \sqrt{\mu} t} & s \geq r .\end{cases}
$$

One then has:

$$
\int_{-1}^{0} d \mu \int_{0}^{t} d r R(r, r, \mu)=\left[\ln \frac{\sqrt{\mu}}{\sinh i v \sqrt{\mu} t}\right]_{-1}^{0} ;
$$

the above formula, when evaluated in $\mu=-1$, produces an extra $i$ factor which cancels the one in the argument of sinh. Substituting this result into Eq. (82), one finds that:

$$
u(t)=\frac{\sinh v t}{v t} .
$$

Substituting Eq. (130) into (75) the prefactor in front of the Green's function becomes:

$$
\sqrt{\frac{\lambda}{\pi v \sinh v t}}
$$

which is the prefactor known in literature [10, 30, 33]. 


\section{EXPONENTIAL CORRELATION FUNCTION}

The results of section III are important by themselves. However, they acquire a further importance when Eqs. (77) and (181) can be solved for some specific, physically meaningful, correlation function. In this section we treat the case in which the correlation function has an exponential damping, i.e.

$$
D(t, s)=\frac{\gamma}{2} e^{-\gamma|t-s|}
$$

where $\gamma$ is the inverse of the correlation time. This type of correlation function was first considered in [17]. Since $D(t, s)$ is time translation invariant, we can use the expression (91) for $z(s)$. The equation for $f(s)$ reads:

$$
f^{\prime \prime}(s)-\frac{i \gamma \omega^{2}}{2} \int_{0}^{t} d r e^{-\gamma|s-r|} f(r)=0
$$

with $\omega$ defined (114). The equation has to be solved with the boundary conditions $f(0)=1, f(t)=$ 0. The solution can be found using the following mathematical procedure [34]: one differentiates the equation twice, obtaining:

$$
f^{\prime \prime \prime \prime}(s)+i \gamma^{2} \omega^{2} f(s)+\gamma^{2}\left(-\frac{i \gamma \omega^{2}}{2} \int_{0}^{s} d l e^{-\gamma(s-l)} f(l)-\frac{i \gamma \omega^{2}}{2} \int_{s}^{t} d l e^{\gamma(s-l)} f(l)\right)=0
$$

next, using once again Eq. (133), one replaces the third term with $-\gamma^{2} f^{\prime \prime}(s)$. In this way Eq. (133) is transformed into the following fourth-order differential equation:

$$
f^{\prime \prime \prime \prime}(s)-\gamma^{2} f^{\prime \prime}(s)+i \gamma^{2} \omega^{2} f(s)=0
$$

The general solution of this equation, which has the advantage of not having an integral term, is easily found and can be expressed as a sum of hyperbolic sines and cosines as follows:

$$
f(s)=f_{1} \sinh v_{1} s+f_{2} \sinh v_{2} s+f_{3} \cosh v_{1} s+f_{4} \cosh v_{2} s,
$$

where $f_{i}$ are constants, to be determined by the boundary conditions, while $v_{i}$ are the two nonsymmetric roots of the bi-quadratic characteristic polynomial associated to Eq. (135), i.e.

$$
v_{1,2}=\sqrt{\frac{1}{2}\left(\gamma^{2} \pm \zeta\right)}, \quad \zeta=\sqrt{\gamma^{4}-4 i \gamma^{2} \omega^{2}}
$$

the other two solutions are: $v_{3}=-v_{1}$ and $v_{4}=-v_{2}$. It is easy to verify that in the white noise limit $(\gamma \rightarrow \infty): v_{1} \rightarrow O(\gamma)$ and $v_{2} \rightarrow v$ defined in (114). 
Since Eq. (135) is a fourth-order differential equation, to determine a specific solution we need four boundary conditions. Two of them are $f(0)=1$ and $f(t)=0$. In order to find the other two conditions, we proceed as in [34]. We evaluate Eq. (133) in 0 and in $t$ :

$$
\left\{\begin{array}{l}
f^{\prime \prime}(0)-\frac{i \gamma \omega^{2}}{2} \int_{0}^{t} d l e^{-\gamma l} f(l)=0 \\
f^{\prime \prime}(t)-\frac{i \gamma \omega^{2}}{2} \int_{0}^{t} d l e^{-\gamma(t-l)} f(l)=0
\end{array}\right.
$$

and we replace $f(l)$ in each expression, using Eq. (135):

$$
\left\{\begin{array}{l}
f^{\prime \prime}(0)+\frac{1}{2 \gamma} \int_{0}^{t} d l e^{-\gamma l}\left(f^{\prime \prime \prime \prime}(l)-\gamma^{2} f^{\prime \prime}(l)\right)=0 \\
f^{\prime \prime}(t)+\frac{1}{2 \gamma} \int_{0}^{t} d l e^{-\gamma(t-l)}\left(f^{\prime \prime \prime \prime}(l)-\gamma^{2} f^{\prime \prime}(l)\right)=0 .
\end{array}\right.
$$

Integrating by parts these two equations, after some algebra one finds that the complete set of boundary conditions is:

$$
\left\{\begin{array}{l}
f(0)=1, \quad f^{\prime \prime \prime}(0)=\gamma f^{\prime \prime}(0) \\
f(t)=0, \quad f^{\prime \prime \prime}(t)=-\gamma f^{\prime \prime}(t) .
\end{array}\right.
$$

The solution of this system involves a long calculation and leads to a complicated expression for the coefficients $f_{i}$ in (136). In order to write the solution $f(s)$ in a compact way, we introduce the following new coefficients:

$$
\begin{aligned}
a_{k} & =\gamma v_{k}^{3}\left[v_{k}^{2}+(-1)^{\bar{k}} \zeta\right], \\
b_{k} & =v_{k}^{2}\left[v_{k}^{4}+(-1)^{\bar{k}} \gamma^{2} \zeta\right], \\
c & =v_{1}^{3} v_{2}^{3}, \\
d_{k} & =-\gamma v_{k}^{3} v_{\bar{k}}^{2},
\end{aligned}
$$

where $k=1,2$ and $\bar{k}=2$ if $k=1, \bar{k}=1$ if $k=2$. With the help of these prescriptions, $f(s)$ can be written as follows:

$$
f(s)=f_{t}(s)=\frac{\sum_{k}\left[r_{t}^{k} \sinh v_{k}(t-s)+u_{t}^{k} \cosh v_{k}(t-s)-u_{s}^{k}\right]}{\sum_{k}\left[2 c+r_{t}^{k} \sinh v_{k} t+u_{t}^{k} \cosh v_{k} t\right]},
$$

where:

$$
\begin{aligned}
r_{t}^{k} & =a_{\bar{k}} \cosh v_{\bar{k}} t+b_{\bar{k}} \sinh v_{\bar{k}} t, \\
u_{t}^{k} & =d_{k} \sinh v_{\bar{k}} t-c \cosh v_{\bar{k}} t .
\end{aligned}
$$


Resorting once again to time translation invariance, we know that $h(s)$ takes the form (98), when the particular solution $h^{\mathrm{P}}(s)$ has the form (97). In order to find $h^{\mathrm{P}}(s)$, we have to consider the non-homogeneous equation:

$$
h^{\prime \prime}(s)-\frac{i \gamma \omega^{2}}{2} \int_{0}^{t} d r e^{-\gamma|s-r|} h(s)=-\frac{i \sqrt{\lambda} \hbar}{m} w(s) .
$$

Following the same calculations which brought from Eq. (133) to Eq. (135), one finds the following fourth-order non-homogeneous equation for $h(s)$ :

$$
h^{\prime \prime \prime \prime}(s)-\gamma^{2} h^{\prime \prime}(s)+i \gamma^{2} \omega^{2} h(s)=-\frac{i \sqrt{\lambda} \hbar}{m}\left(w^{\prime \prime}(s)-\gamma^{2} w(s)\right)
$$

A particular solution of this equation, having the form (97), is [35]:

$$
h^{\mathrm{P}}(s)=-\frac{i \sqrt{\lambda} \hbar}{m} \int_{0}^{s} \bar{f}_{s}(l)\left(w^{\prime \prime}(l)-\gamma^{2} w(l)\right) d l,
$$

where $\bar{f}_{s}(l)$ is the solution of Eq. (135), thus having the general form (136), with boundary conditions: $f_{s}(s)=f_{s}^{\prime}(s)=f_{s}^{\prime \prime}(s)=0, f_{s}^{\prime \prime \prime}(s)=1$. After some simple calculations, one finds the following expression:

$$
\bar{f}_{s}(l)=-\frac{1}{\zeta} \sum_{k}(-1)^{k} \frac{\sinh v_{k}(s-l)}{v_{k}} .
$$

We now have all functions which are necessary to compute the Green's function. This takes the form (92), where the coefficients $\mathcal{A}_{t}-\mathcal{E}_{t}$ are defined in Eqs. (93)-(96) in terms of the following functions:

$$
\begin{aligned}
f_{t}^{\prime}(0) & =-\frac{\sum_{k} v_{k}\left(r_{t}^{k} \cosh v_{k} t+u_{t}^{k} \sinh v_{k} t+d_{\bar{k}}\right)}{\sum_{k}\left[2 c+r_{t}^{k} \sinh v_{k} t+u_{t}^{k} \cosh v_{k} t\right]}, \\
f_{t}^{\prime}(t) & =-\frac{\sum_{k} v_{k}\left(r_{t}^{k}+d_{\bar{k}} \cosh v_{k} t-c \sinh v_{k} t\right)}{\sum_{k}\left[2 c+r_{t}^{k} \sinh v_{k} t+u_{t}^{k} \cosh v_{k} t\right]},
\end{aligned}
$$

and:

$$
\begin{aligned}
h^{\mathrm{P}^{\prime}}(0) & =0 \\
h^{\mathrm{P}^{\prime}}(t) & =\frac{i \sqrt{\lambda} \hbar}{m \zeta} \int_{0}^{t} \sum_{k}(-1)^{k} \cosh v_{k}(t-l)\left(w^{\prime \prime}(l)-\gamma^{2} w(l)\right) .
\end{aligned}
$$

In terms of these functions, the entire dynamics of the free particle can be analyzed.

Eq. (81) for $R(s, r, \mu)$ can be solved similar to Eq. (77). One then finds the explicit expression of the prefactor of the Green's function (92). This goes outside the scope of the present paper, so we omit the calculation. 


\section{DENSITY MATRIX EVOLUTION AND IMAGINARY NOISE TRICK}

One of the major difficulties connected to Eq. (6) arises when one wants to compute average values of the form $\mathbb{E}_{\mathbb{P}}\left[\left\langle\psi_{t}|O| \psi_{t}\right\rangle\right] \equiv \mathbb{E}_{\mathbb{Q}}\left[\left\langle\phi_{t}|O| \phi_{t}\right\rangle\right]$, where $\phi_{t}$ solves Eq. (6) and $\psi_{t}$ is the corresponding normalized solution; $O$ is a generic self-adjoint operator. Averages of this kind are particularly important, as they represent physical quantities, directly connected to experimental outcomes. The difficulty in computing such averages lies both in the difficulty in solving Eq. (6) and in the fact that $\phi_{t}$ depends non-trivially on the noise $w(t)$.

In the white noise-case, a very helpful trick, known as the imaginary noise trick [21, 36] allows to simplify considerably the problem. Let us consider the following class of stochastic differential equations:

$$
d \psi_{t}^{\xi}=\left[-\frac{i}{\hbar} H d t+\sqrt{\gamma} \sum_{n=1}^{N}\left(\xi q_{n}-\xi_{\mathrm{R}}\left\langle q_{n}\right\rangle_{t}\right) d W_{n, t}-\frac{\gamma}{2} \sum_{n=1}^{N}\left(|\xi|^{2} q_{n}^{2}-2 \xi \xi_{\mathrm{R}} q_{n}\left\langle q_{n}\right\rangle_{t}+\xi_{\mathrm{R}}^{2}\left\langle q_{n}\right\rangle_{t}^{2}\right) d t\right] \psi_{t}^{\xi}
$$

where $\xi=\xi_{\mathrm{R}}+i \xi_{\mathrm{I}}$ is a constant complex factor. By using standard Itô calculus one can show that $\mathbb{E}_{\mathbb{P}}\left[\left\langle\psi_{t}^{\xi}|O| \psi_{t}^{\xi}\right\rangle\right]$ depends only on $|\xi|^{2}$ : there is, so to speak, an invariance under phase change in the coupling constant. When $\xi=1$ one recovers Eq. (1), while when $\xi=i$, one obtains a standard Schrödinger equation with a stochastic potential, generating a unitary (thus non-collapsing) evolution, without non-linear terms; this equation is much simpler to analyze than Eq. (11), nevertheless, at the statistical level, it gives the same results as Eq. (11) does.

In 21] it has been shown that the imaginary noise trick holds also in the non-white noise case, at least to second order in the perturbation expansion with respect to the parameter $\sqrt{\lambda}$. Aim of this section is to prove that this is an exact property which holds to all orders.

Let us consider the following class of equations:

$$
\frac{d}{d t} \phi_{t}^{\xi}(x)=\left[-\frac{i}{\hbar} H+\xi \sqrt{\lambda} q w(t)-2 \xi_{\mathrm{R}} \sqrt{\lambda} q \int_{0}^{t} d s D(t, s) \frac{\delta}{\delta w(s)}\right] \phi_{t}^{\xi}(x)
$$

obviously, when $\xi=1$ one recovers Eq. (53) for a free particle. Since $\mathbb{E}_{\mathbb{P}}\left[\left\langle\psi_{t}|O| \psi_{t}\right\rangle\right] \equiv$ $\mathbb{E}_{\mathbb{Q}}\left[\left\langle\phi_{t}|O| \phi_{t}\right\rangle\right] \equiv \operatorname{Tr}\left[O \rho_{t}\right]$ where the density matrix $\rho_{t}$ is defined as: $\rho_{t}:=\mathbb{E}_{\mathbb{P}}\left[\left|\psi_{t}\right\rangle\left\langle\psi_{t}\right|\right] \equiv \mathbb{E}_{\mathbb{Q}}\left[\left|\phi_{t}\right\rangle\left\langle\phi_{t}\right|\right]$, in order to prove the required property it is sufficient to show that $\rho_{t}$ depends only on $|\xi|^{2}$. The propagator associated to Eq. (157) reads

$$
G_{\xi}\left(x, t ; x_{0}, 0\right)=\int_{q(0)=x_{0}}^{q(t)=x} \mathcal{D}[q] e^{\mathcal{S}_{\xi}[q]}
$$

where

$$
\mathcal{S}_{\xi}[q]=\int_{0}^{t} d s\left[\frac{i}{\hbar} \mathcal{S}_{0}[q]+\xi \sqrt{\lambda} q(s) w(s)-\xi_{\mathrm{R}} \xi \lambda q(s) \int_{0}^{t} d r q(r) D(s, r)\right]
$$


and $\mathcal{S}_{0}[q]$ is the standard action associated to the quantum Hamiltonian $H$. The propagator $J\left(x, x^{\prime}, t ; x_{0}, x_{0}^{\prime}, 0\right)$ associated to $\rho_{t}\left(x, x^{\prime}\right):=\left\langle x\left|\rho_{t}\right| x^{\prime}\right\rangle$ can then be expressed as follows [28]:

$$
J\left(x, x^{\prime}, t ; x_{0}, x_{0}^{\prime}, 0\right)=\mathbb{E}_{\mathbb{Q}}\left[G_{\xi}\left(x, t ; x_{0}, 0\right) G_{\xi}^{*}\left(x^{\prime}, t ; x_{0}^{\prime}, 0\right)\right],
$$

where $^{*}$ denotes complex conjugation. Substituting the definition (158) for the wave function's propagator into (160), and exchanging the path-integration with the stochastic average, the stochastic terms average as follows:

$$
\begin{aligned}
& \mathbb{E}_{\mathbb{Q}}\left[\exp \left[\int_{0}^{t} d s\left(\xi \sqrt{\lambda} q(s)+\xi^{*} \sqrt{\lambda} q^{\prime}(s)\right) w(s)\right]\right]= \\
& =\exp \left[\frac{1}{2} \int_{0}^{t} d s \int_{0}^{t} d r D(s, r)\left(\xi^{2} \lambda q(s) q(r)+\xi^{* 2} \lambda q^{\prime}(s) q^{\prime}(r)+|\xi|^{2} \lambda q(s) q^{\prime}(r)+|\xi|^{2} \lambda q(r) q^{\prime}(s)\right)\right] .
\end{aligned}
$$

The propagator then becomes:

$$
\begin{aligned}
J\left(x, x^{\prime}, t ; x_{0}, x_{0}^{\prime}, 0\right)=\int_{q(0)=x_{0}}^{q(t)=x} \mathcal{D}[q] & \int_{q^{\prime}(0)=x_{0}^{\prime}}^{q^{\prime}(t)=x^{\prime}} \mathcal{D}\left[q^{\prime}\right] \exp \left[\int _ { 0 } ^ { t } d s \left(\frac{i}{\hbar} \mathcal{S}_{0}[q]-\frac{i}{\hbar} \mathcal{S}_{0}\left[q^{\prime}\right]\right.\right. \\
& \left.\left.-\frac{\lambda}{2}|\xi|^{2} \int_{0}^{t} d r D(s, r)\left(q(s)-q^{\prime}(s)\right)\left(q(r)-q^{\prime}(r)\right)\right)\right],
\end{aligned}
$$

which depends only on $|\xi|^{2}$. We can conclude that the evolution for the density matrix is independent from a phase change in the coupling with the noise.

\section{A. Average values of physical quantities}

As anticipated, this phase change invariance provide a very handy tool to compute average values, such as the mean position and mean momentum. In place of Eq. (53), let us consider the equation:

$$
\frac{d}{d t} \phi_{t}(x)=\left[-\frac{i}{\hbar} \frac{p^{2}}{2 m}+i \sqrt{\lambda} q w(t)\right] \phi_{t}(x)
$$

which belongs to the class (157), with $\xi=i$. This is a linear, unitary, norm-preserving standard Schrödinger equation for a free particle under the influence of a stochastic potential. The evolution of the stochastic average of the mean value of an observable $O$ is given by the following equation:

$$
\frac{d}{d t} \mathbb{E}_{\mathbb{Q}}\left[\langle O\rangle_{t}\right]=\frac{i}{2 m \hbar} \mathbb{E}_{\mathbb{Q}}\left[\left\langle\left[p^{2}, O\right]\right\rangle_{t}\right]-i \sqrt{\lambda} \mathbb{E}_{\mathbb{Q}}\left[w(t)\langle[q, O]\rangle_{t}\right]
$$

where, as usual, $\langle\cdot\rangle_{t}=\left\langle\phi_{t}|\cdot| \phi_{t}\right\rangle$. 
The mean in position and momentum can now be easily computed. Remembering that $\left\langle\phi_{t} \mid \phi_{t}\right\rangle=$ 1 and that the mean of $w(t)$ is 0 , one finds:

$$
\begin{aligned}
\frac{d}{d t} \mathbb{E}_{\mathbb{Q}}\left[\langle q\rangle_{t}\right] & =\frac{1}{m} \mathbb{E}_{\mathbb{Q}}\left[\langle p\rangle_{t}\right], \\
\frac{d}{d t} \mathbb{E}_{\mathbb{Q}}\left[\langle p\rangle_{t}\right] & =0 ;
\end{aligned}
$$

as we see, we recover Newton's equations for a free particle. In particular, also in the non-Markovian case, like in the white-noise case, the momentum of an isolated system is conserved, in the average. In sec.VIII, where we analyze the time evolution of Gaussian states, we will see that the fluctuations around the average are inversely proportional to the mass of the system. These two facts, together with the fact that the collapse scales with the size of the system, lead to the following result: the wave function of an isolated macro-object behaves, for all practical purposes, like a particle moving deterministically in space according to Newton's laws.

Because of the many experimental implications [13], it is important to check how the mean free energy $H_{0}=p^{2} / 2 m$ evolves in time. This is most easily computed by shifting to the Heisenberg picture, in which case one finds [21]:

$$
\frac{d}{d t} \mathbb{E}_{\mathbb{Q}}\left[\left\langle H_{0}\right\rangle_{t}\right]=\frac{\lambda \hbar^{2}}{m} \int_{0}^{t} d s D(t, s)
$$

this is the expected generalization of the well-known white noise formula for the mean energy increase in collapse models [9, 12, 14]. For a physically reasonable correlation function such as the exponential one of Eq. (132), one has:

$$
\mathbb{E}_{\mathbb{Q}}\left[\left\langle H_{0}\right\rangle_{t}\right]=\left\langle H_{0}\right\rangle_{0}+\frac{\lambda \hbar^{2}}{2 m}\left(t+\frac{e^{-\gamma t}-1}{\gamma}\right):
$$

as we see, also in this case the mean energy increases linearly in time, without reaching a steady value. More generally, let us assume time translation invariance, and let us consider the spectral decomposition of a generic correlator $D(|t-s|)$ :

$$
D(|t-s|)=\int_{0}^{\infty} d \omega \tilde{D}(\omega) \cos \omega(t-s)
$$

we have:

$$
\int_{0}^{t} d s D(t, s)=\int_{0}^{\infty} \frac{d u}{u} \tilde{D}(u / t) \sin u \underset{t \rightarrow+\infty}{\longrightarrow} \frac{\pi}{2} \tilde{D}(0)
$$

The above formula shows that the energy production is nonzero also at large times, unless the correlator has a cutoff at low frequencies [21]. 
An important lesson to learn from the above analysis is that non-Markovian terms do not introduce thermalization effects in the evolution of a quantum system; such effects can be introduced only by modifying the form of the operator coupled to the noise, as first discussed in [37]. Nevertheless, non-Markovian terms are extremely important, as they affect the time evolution of physical quantities, thus the predictions of collapse models, at small times. A significative example has been first provided in [38].

\section{EVOLUTION OF A GAUSSIAN WAVE FUNCTION}

In this section we study the time evolution of Gaussian wave functions, whose form is clearly preserved by the Green's function (86); for simplicity, we will assume time translation invariance, so that (86) reduces to (92). A generic Gaussian state of the form:

$$
\phi_{0}\left(x_{0}\right)=\exp \left[-\alpha_{0} x_{0}^{2}+\beta_{0} x_{0}+\gamma_{0}\right]
$$

evolves in time to the following state:

$$
\phi_{t}(x)=\exp \left[-\alpha_{t} x^{2}+\beta_{t} x+\gamma_{t}\right]
$$

where:

$$
\begin{aligned}
\alpha_{t} & =\mathcal{A}_{t}-\frac{\mathcal{B}_{t}^{2}}{4\left(\alpha_{0}+\mathcal{A}_{t}\right)}, \\
\beta_{t} & =-\frac{\mathcal{C}_{t}+\beta_{0}}{2\left(\alpha_{0}+\mathcal{A}_{t}\right)} \mathcal{B}_{t}+\mathcal{D}_{t}, \\
\gamma_{t} & =\gamma_{0}+\mathcal{E}_{t}+\frac{\left(\mathcal{C}_{t}+\beta_{0}\right)^{2}}{4\left(\alpha_{0}+\mathcal{A}_{t}\right)},
\end{aligned}
$$

and the functions $\mathcal{A}_{t}-\mathcal{E}_{t}$ have been defined in Eqs. (93)-(96). We can draw some important conclusions about the time evolution of Gaussian wave functions:

- Since neither $\mathcal{A}_{t}$ nor $\mathcal{B}_{t}$ depend on the noise $w(t)$, the time evolution of the spread of the wave function is deterministic, as in the white noise case [9]. An initially spread-out Gaussian wave function shrinks in space, reaching an asymptotic final spread. In the case of an exponential correlation function, we can provide the explicit expression for the asymptotic value for the spread (see next subsection).

- By making the substitution:

$$
x \rightarrow y:=\sqrt{\frac{m}{m_{0}}} x,
$$


one can easily prove that Eq. (53), thus the propagator (92), does not depend on the mass $m$ of the particle. Thus, one way to see the effect of the mass on the global dynamics is to take the evolution of the wave function for the reference mass $m_{0}$, and then "shrink" the space coordinates by a factor $\sqrt{m_{0} / m}$. This leads to the amplification mechanism, which is the characteristic feature of collapse models: the bigger the system, the faster the wave function shrinks in space.

- The center $\langle q\rangle_{t}=\beta_{t}^{\mathrm{R}} / 2 \alpha_{t}^{\mathrm{R}}$ of the Gaussian wave function (172) evolves randomly in space, as expected. Its average value has already been computed in (165), and evolves classically. Moreover, due to the independence of the dynamics from $m$ under the substitution (176), one can conclude that the fluctuations of $\langle q\rangle_{t}$ around the classical motion go like $m^{-1 / 2}$ since the following equality for the variance $\mathbb{V}_{q}:=\sqrt{\mathbb{E}_{\mathbb{Q}}\left[\langle q\rangle_{t}-\mathbb{E}_{\mathbb{Q}}\left[\langle q\rangle_{t}\right]\right]^{2}}$ follows immediately from (176):

$$
\mathbb{V}_{q}^{m}=\sqrt{\frac{m_{0}}{m}} \mathbb{V}_{q}^{m_{0}}
$$

where the apex $m$ indicates with respect to which mass the variance is computed. Eq. (177) in agreement with the white noise case. This means that the bigger the system, the smaller the fluctuations: in the microscopic case, in this way, one recovers classical determinism for all pratical pourposes.

- The mean momentum $\langle p\rangle_{t}=\hbar\left[\beta_{t}^{\mathrm{I}}-\left(\alpha_{t}^{\mathrm{I}} / \alpha_{t}^{\mathrm{R}}\right) \beta_{t}^{\mathrm{R}}\right]$ also evolves randomly in space. Its average value is constant in time (see Eq. (166)), as expected from a free particle, while the fluctuations around the average increase like $m^{1 / 2}$. If however we consider the fluctuations of the mean velocity, we have that they decrease like $m^{-1 / 2}$. Thus also in this respect one recovers classical determinism at the macroscopic level.

The above remarks show that the non-Markovian QMUPL model shares all the important features of the white noise model. More quantitative details can be given by taking a specific expression for the correlation function $D(t, s)$, as we will do in the next subsection.

\section{A. Exponential correlation function}

We study in some more detail the time evolution of the Gaussian solution (172), when the correlation function is exponential as in (132). We focus our attention on the spread in position $\sigma(t)$ which is given by the inverse of twice the square root of the real part of $\alpha_{t}$, whose analytic 


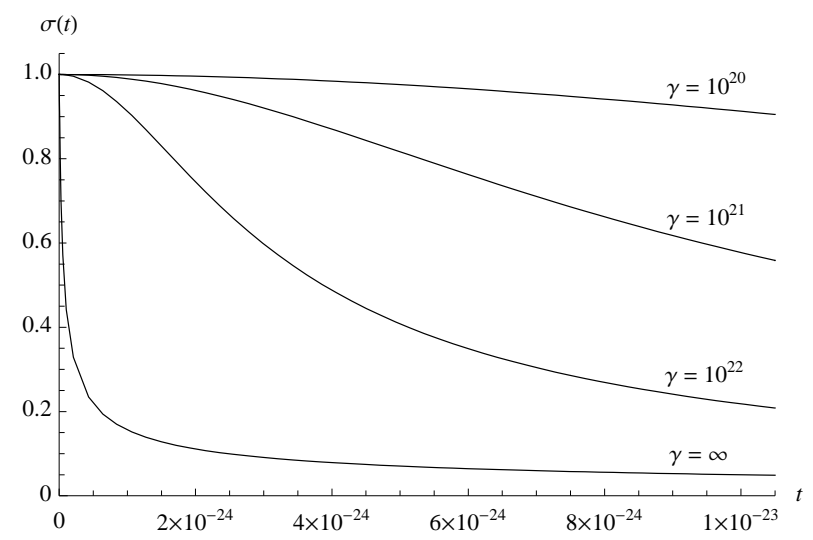

FIG. 1: Linear/linear graph showing the time evolution of $\sigma(t)$ for a $1 \mathrm{Kg}$ particle, for different values of $\gamma$, for the same initial condition $\sigma(0)=1$ and with $\lambda_{0}=5.00 \times 10^{-3} \mathrm{~m}^{-2} \mathrm{sec}^{-1}$. The curve with $\gamma=\infty$ corresponds to the white noise case. Time is measured in seconds, distances in meters.

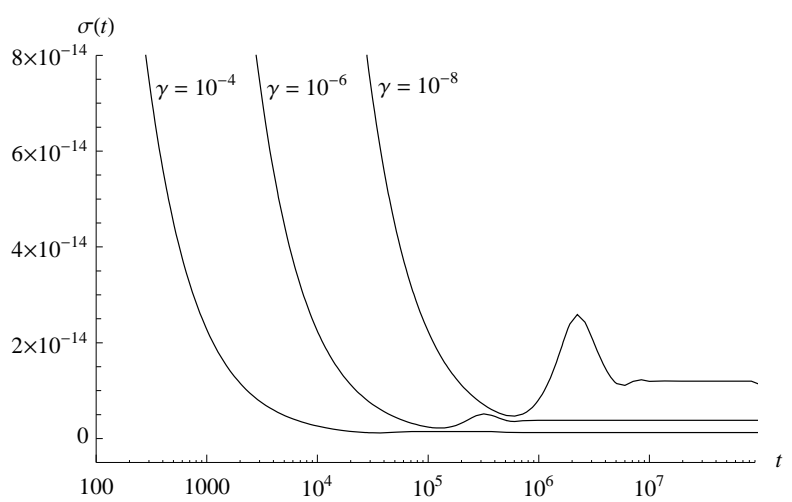

FIG. 2: Log/linear graph, showing the large time behavior of $\sigma(t)$ for a $1 \mathrm{Kg}$ particle, for different values of $\gamma$, for the same initial condition $\sigma(0)=1$ and with $\lambda_{0}=5.00 \times 10^{-3} \mathrm{~m}^{-2} \mathrm{sec}^{-1}$. The white noise case would appear in the graph as a straight line with value $1.27 \times 10^{-15} \mathrm{~m}$. Time is measured in seconds, distances in meters.

expression is given explicitly by Eqs. (173), (93) and (152). Figs. 1 and 2 show some typical behavior of $\sigma(t)$ for small times (Fig. 1) and large times (Fig. 2), for different values of $\gamma$ and for $m=1 \mathrm{Kg}$. As we see and as we expect, the larger the value of $\gamma$, the stronger the noise and the faster the collapse in space.

Fig. 3 shows how the spread of a Gaussian wave function associated to a particle having a mass $m=1.01 \times 10^{-3} \mathrm{Kg}$ (which is the total mass of a system of $6.02 \times 10^{23}$ nucleons), having initial spread $\sigma(0)=1 \mathrm{~m}$, decreases after $10^{-3}$ sec, as a function of $\gamma$. The value of the coupling constant 


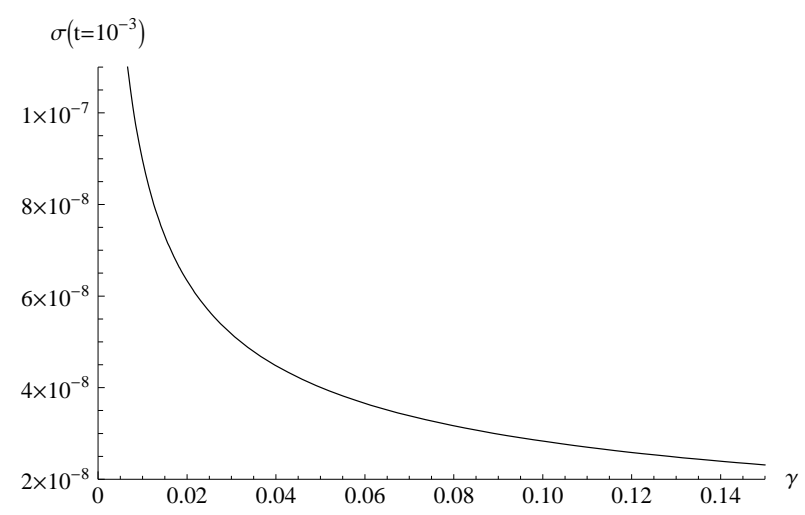

FIG. 3: Linear/linear graph, showing to which value an initial spread $\sigma(0)=1 \mathrm{~m}$ decreases after $10^{-3}$ sec, as a function of $\gamma$. The mass of the particle has been set equal to $m=1.01 \times 10^{-3} \mathrm{Kg}$, corresponding to the total mass of a system containing an Avogadro's number of nucleons. The coupling constant $\lambda_{0}$ has been given the GRW-value $5.00 \times 10^{-3} \mathrm{~m}^{-2} \mathrm{sec}^{-1}$. Larger values of $\gamma$ imply a faster collapse in space. Time is measured in seconds, distances in meters.

$\lambda_{0}$ is the GRW value (3). GRW have chosen the value of the coupling constant in order to ensure that the wave function of a system of at least an Avogadro's number of particle collapses, within a time interval of $10^{-3}$ sec, below $10^{-7} \mathrm{~m}$. Fig. 3 shows that also for relatively small values of $\gamma$, the non-Markovian collapse models preserves this same feature of the white-noise model.

Fig. 4 displays the same graph as that of Fig. 3, with the typical values chosen by Adler. In 13] Adler sets the value of the CSL-coupling constant $\gamma_{\mathrm{CSL}}$ equal to $2 \times 10^{-21 \pm 2} \mathrm{~cm}^{3} \mathrm{sec}^{-1}$ by noting that in the process of latent image formation, which has a characteristic time of about $3.33 \times 10^{-2 \pm 2}$ sec, a number of atoms approximately equal to 20 is involved. He then assumes that the collapse process ensures that the reduction occurs already at this stage, from which the value $\gamma_{\mathrm{CSL}}=2 \times 10^{-21 \pm 2} \mathrm{~cm}^{3} \mathrm{sec}^{-1}$ comes. The graph shows that even for relatively small values of $\gamma$ (Adler and Ramazanoglu [38] have shown that choosing $\gamma \sim 10^{15} \mathrm{sec}^{-1}$ already changes significantly the predictions of non-Markovian models with respect to white-noise models), an initially spreadout wave function shrinks rapidly below $10^{-7} \mathrm{~m}$, which corresponds to a well-localized wave packet.

For an exponential correlation function, we can write down the analytic expression of the asymptotic value of $\alpha_{t}$, which is:

$$
\alpha_{\infty}=\lim _{t \rightarrow \infty} \alpha_{t}=-\frac{i m}{2 \hbar}\left(v_{1}+v_{2}-\gamma\right)
$$

with $v_{1,2}$ defined in (137). From this, the asymptotic spread in position can be easily computed. 


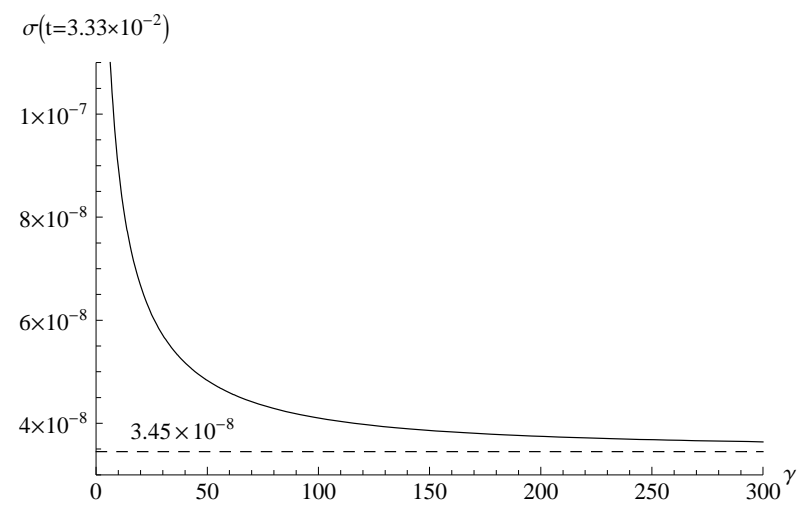

FIG. 4: Linear/linear graph, showing to which value an initial spread $\sigma(0)=1 \mathrm{~m}$ decreases after $3.33 \times 10^{-2}$ sec, as a function of $\gamma$. The mass of the particle has been set equal to $m=1.06 \times 10^{-18} \mathrm{Kg}$, which is the mass of a system of $n^{2} N(n=5640, N=20)$ nucleons, with $n$ and $N$ taken from Eq. (8) of [13]. The coupling constant $\lambda_{0}$ has been given Adler's middle value $1.12 \times 10^{6} \mathrm{~m}^{-2} \mathrm{sec}^{-1}$. The dashed line corresponds to the white noise case. Time is measured in seconds, distances in meters.

In particular, if we take the white noise limit $\gamma \rightarrow \infty$, one obtains the finite value:

$$
\alpha_{\infty}^{\mathrm{WN}}=\sqrt{\frac{\lambda m}{2 i \hbar}}
$$

which matches with the value known in the literature [9].

\section{CONCLUSIONS}

We have thoroughly investigated the dynamics of a free quantum particle as described by the non-Markovian QMUPL model of spontaneous wave function collapse. We have provided an explicit formula for the Green's function; we have shown that it reproduces the well-known white noise case, and have analyzed the physically interesting case of an exponential correlation function. We have proven that the non-Markovian model shares all the most important features of the corresponding white-noise model; we have described in particular the evolution of Gaussian wave functions.

There are of course several other important issues which need to be investigated. In particular:

- It is important to set on a rigorous mathematical ground the change of measure defined in (9), and to derive the analogous of Girsanov's theorem which holds for the white-noise case. A sketch of the proof can be found in Appendix B of Ref. [17].

- It is also important to set a bound on the spread of the general solution as a function of time, in order to see how it decays in time. Such a formula would be crucial for setting a lower 
bound on the value of $\lambda$ by guaranteeing that the model collapses sufficiently big systems in sufficiently short time.

- In the case of an exponential correlation function, it would be interesting to prove if any initial state collapses asymptotically to a Gaussian state whose spread in position and momentum is fixed and given by (178). In this paper we have proved that this is true only for the special case of initially Gaussian states. A similar general theorem has been recently proved for the white-noise case [10].

These problems will be the subject of future investigation.

\section{Acknowledgments}

The authors wish to thank S.L. Adler, D. Dürr and G.C. Ghirardi for many useful conversations. They also wish to thank P. Pearle for showing them that the Green's function can be equally well computed by using the standard operator formalism in place of the path integral formalism. They finally wish to thank A. Fonda for his guidance through the proof of the existence and uniqueness theorem of Appendix A

\section{APPENDIX A: AN EXISTENCE AND UNIQUENESS THEOREM}

In this appendix we prove that equation (777):

$$
\frac{i m}{2 \hbar} z^{\prime \prime}(s)+\lambda \int_{0}^{t} d r D(s, r) z(r)=\frac{\sqrt{\lambda}}{2} w(s)
$$

with boundary conditions $z(0)=x_{0}, z(t)=x$, admits a unique solution. The same theorem applies also to Eq. (81) for $R(s, r, \mu)$. In order to simplify the proof, it is convenient to set both boundary conditions to zero. This can be done without loss of generality, as follows. Let us define the new function:

$$
\bar{z}(s)=z(s)-y(s), \quad y(s):=\frac{x-x_{0}}{t} s+x_{0} ;
$$

obviously, $\bar{z}(0)=\bar{z}(t)=0$. Moreover, $\bar{z}(s)$ solves the following equation:

$$
\frac{i m}{2 \hbar} \bar{z}^{\prime \prime}(s)+\lambda \int_{0}^{t} d r D(s, r) \bar{z}(r)=\frac{\sqrt{\lambda}}{2} \bar{w}(s)
$$

where

$$
\bar{w}(s)=w(s)-2 \sqrt{\lambda} \int_{0}^{t} d r D(s, r) y(r) .
$$


It is then sufficient to prove the following theorem.

Theorem. Let $D(t, s)$ be a real continuous function on $[0, t] \times[0, t]$, symmetric in its two variables; let $f(s)$ belong to $C[0, t]$. Then the integro-differential equation

$$
i z^{\prime \prime}(s)+\int_{0}^{t} d r D(s, r) z(r)=f(s),
$$

with boundary conditions $z(0)=z(t)=0$, admits a unique solution $z(s) \in C^{2}[0, t]$.

Proof. Let $\mathcal{D}(D) \subset C^{2}[0, t]$ the set of functions $z(s) \in C^{2}[0, t]$ such that $z(0)=z(t)=0$. Define the following operators:

$$
\begin{aligned}
D: \mathcal{D}(D) \rightarrow C[0, t], & D[z(s)] & =i z^{\prime \prime}(s), \\
I: C[0, t] \rightarrow C[0, t], & I[z(s)] & =\int_{0}^{t} d r D(s, r) z(r) ;
\end{aligned}
$$

with these definitions Eq. (A5) becomes

$$
D[z(s)]+I[z(s)]=f(s) .
$$

The integral operator $I$ is compact (see, e.g., Theorem 8.7-5, page 454 of [39]), while $D$ is invertible and its inverse reads:

$$
\begin{aligned}
D^{-1} & : C[0, t] \rightarrow \mathcal{D}(D), \\
D^{-1}[g(s)] & =-i\left(\int_{0}^{s} d u \int_{0}^{u} d v g(v)-\frac{s}{t} \int_{0}^{t} d u \int_{0}^{u} d v g(v)\right) .
\end{aligned}
$$

Since it has an integral form, also $D^{-1}$ is compact. We can write Eq. (A8) as follows:

$$
\left(\operatorname{Id}-D^{-1} I\right)[z(s)]=\tilde{f}(s), \quad z(0)=z(t)=0,
$$

where Id is the identity operator and $\tilde{f}(s)=D^{-1} f(s) \in C^{2}[0, t]$.

Our problem reduces to showing existence and uniqueness of solutions for Eq. (A11). Since the product of two compact operators is compact, then also $D^{-1} I$ is compact. We now use Fredholm's Alternative Theorem (Theorem 8.7-2, page 452 of [39]), according to which in order to prove the theorem it suffices to show that the homogeneous equation associated to Eq. (A11), i.e.

$$
\left(\operatorname{Id}-D^{-1} I\right)[z(s)]=0, \quad z(0)=z(t)=0,
$$

admits only the trivial solution $z(s)=0$.

To prove this, we separate the real and imaginary part of $z(s)$ which we respectively denote with the superscripts R,I, obtaining

$$
\begin{aligned}
& \left(z^{\mathrm{R}}\right)^{\prime \prime}(s)+\int_{0}^{t} d r D(s, r) z^{\mathrm{I}}(r)=0, \\
& \left(z^{\mathrm{I}}\right)^{\prime \prime}(s)-\int_{0}^{t} d r D(s, r) z^{\mathrm{R}}(r)=0,
\end{aligned}
$$


with boundary conditions $z^{\mathrm{R}}(0)=z^{\mathrm{R}}(t)=z^{\mathrm{I}}(0)=z^{\mathrm{I}}(t)=0$. Multiplying these two equations respectively by $z^{\mathrm{R}}(s)$ and $z^{\mathrm{I}}(s)$, and integrating by parts one finds:

$$
\begin{aligned}
& \int_{0}^{t} d s\left[\left(z^{\mathrm{R}}\right)^{\prime}(s)\right]^{2}-\int_{0}^{t} d s z^{\mathrm{R}}(s) \int_{0}^{t} d r D(s, r) z^{\mathrm{I}}(r)=0, \\
& \int_{0}^{t} d s\left[\left(z^{\mathrm{I}}\right)^{\prime}(s)\right]^{2}+\int_{0}^{t} d s z^{\mathrm{I}}(s) \int_{0}^{t} d r D(s, r) z^{\mathrm{R}}(r)=0 .
\end{aligned}
$$

Exploiting the symmetry of $D(s, r)$ in its variables, we can sum the two equations, obtaining:

$$
\int_{0}^{t} d s\left[\left(\left(z^{\mathrm{R}}\right)^{\prime}(s)\right)^{2}+\left(\left(z^{\mathrm{I}}\right)^{\prime}(s)\right)^{2}\right]=0 ;
$$

this implies that $z^{\mathrm{R}}(s)$ and $z^{\mathrm{I}}(s)$ are constants and, applying the boundary conditions, these constants are equal to zero. We can then state that Eq. (A12) admits only the trivial solution $z(s)=0$. This proves existence and uniqueness of solutions for that Eq. (A11), and thus for Eq. (77).

[1] L. Diósi, Models for universal reduction of macroscopic quantum fluctuations, Phys. Rev. A 40, 1165 (1989).

[2] L. Diósi, Relativistic theory for continuous measurement of quantum fields, Phys. Rev. A 42, 5086 (1990).

[3] V. P. Belavkin and P. Staszewski, A quantum particle undergoing continuous observation, Phys. Lett. A 140, 359 (1989).

[4] V. P. Belavkin and P. Staszewski, Nondemolition observation of a free quantum particle, Phys. Rev. A 45, 1347 (1992).

[5] D. Chruściński and P. Staszewski, On the asymptotic solutions of Belavkin's stochastic wave equation, Phys. Scripta 45, 193 (1992).

[6] D. Gạtarek and N. Gisin, Continuous quantum jumps and infinite-dimensional stochastic equations, J. Math. Phys. 32, 2152 (1991).

[7] J. Halliwell and A. Zoupas, Quantum state diffusion, density matrix diagonalization, and decoherent histories: a model, Phys. Rev. D 52, 7294 (1995).

[8] A. S. Holevo, On dissipative stochastic equations in a Hilbert space, Probab. Theory Relat. Fields 104, 483 (1996).

[9] A. Bassi, Collapse models: analysis of the free particle dynamics, J. Phys. A 38, 3173 (2005).

[10] A. Bassi, D. Dürr and M. Kolb, On the long time behavior of stochastic schroedinger evolutions, preprint arXiv:0811.1877.

[11] A. Bassi and D. Dür, On the long time behavior of hilbert space diffusion, Europhys. Lett. 84, 10005 (2008).

[12] G. C. Ghirardi, A. Rimini and T. Weber, Unified dynamics for microscopic and macroscopic systems, Phys. Rev. D 34, 470 (1986). 
[13] S. L. Adler, Lower and upper bounds on CSL parameters from latent image formation and IGM heating, J. Phys. A 40, 2935 (2007).

[14] G. C. Ghirardi, P. Pearle and A. Rimini, Markov processes in hilbert space and continuous spontaneous localization of systems of identical particles, Phys. Rev. A 42, 78 (1990).

[15] R. S. Liptser and A. N. Shiryaev, Statistics of random processes. I general theory, volume 5 of Applications of Mathematics, Springer-Verlag, Berlin (2001).

[16] L. Diósi and W. T. Strunz, The non-Markovian stochastic Schrödinger equation for open systems, Phys. Lett. A 235, 569 (1997).

[17] L. Diósi, N. Gisin and W. T. Strunz, Non-Markovian quantum state diffusion, Phys. Rev. A 58, 1699 (1998).

[18] A. Bassi and G. C. Ghirardi, Dynamical reduction models with general gaussian noises, Phys. Rev. A 65, $042114(2002)$.

[19] I. I. Gikhman and A. V. Skorokhod, Introduction to the theory of random processes, Dover Publications Inc., Mineola, NY (1996).

[20] T. Yu, L. Diosi, N. Gisin and W. T. Strunz, Non-Markovian quantum-state diffusion: perturbation approach, Phys. Rev. A 60, 91 (1999).

[21] S. L. Adler and A. Bassi, Collapse models with non-white noises, J. Phys. A 40, 15083 (2007).

[22] S. L. Adler and A. Bassi, Collapse models with non-white noises ii: particle-density coupled noises, J. Phys. A 41, 395308 (2008).

[23] R. P. Feynman and Jr. F.L Vernon, The theory of a general quantum system interacting with a linear dissipative system, Ann. Phys. 24, 118 (1963).

[24] P. Pearle, in Perspectives on quantum reality, Kluwer Acad. Publ., Dordrecht (1996).

[25] P. Pearle, in Open systems and measurement in relativistic quantum theory, Springer-Verlag, Berlin (1999).

[26] W. T. Strunz, Linear quantum state diffusion for non-Markovian open quantum systems, Phys. Lett. A 224, 25 (1996).

[27] D. S. Bernstein, Matrix mathematics, Princeton University Press, Princeton, NJ (2005).

[28] R. P. Feynman and A. R. Hibbs, Quantum mechanics and path integrals, McGraw-Hill, New York (1965).

[29] D. C. Khandekar, S. V. Lawande and K. V. Bhagwat, Path integration of a two-time quadratic action, J. Phys. A 16, 4209 (1983).

[30] C. Grosche and F. Steiner, Handbook of Feynman path integrals, Springer Verlag, Berlin (1998).

[31] H. J. W. Müller-Kirsten, Introduction to quantum mechanics: Schrödinger equation and path integral, World Scientific, Hackensack, NJ (2006).

[32] A. D. Polyanin and V. F. Zaitsev, Handbook of exact solutions for ordinary differential equations, Chapman \& Hall/CRC, Boca Raton, FL (2003).

[33] V. N. Kolokol'tsov, Localization and analytic properties of the solutions of the simplest quantum filtering 
equation, Rev. Math. Phys. 10, 801 (1998).

[34] A. D. Polyanin and A. V. Manzhirov, Handbook of integral equations, Chapman \& Hall/CRC, Boca Raton, FL (2008).

[35] P. Hartman, Ordinary differential equations, SIAM, Philadelphia, PA (2002).

[36] G. C. Ghirardi, R. Grassi and P. Pearle, Relativistic dynamical reduction models: general framework and examples, Found. Phys. 20, 1271 (1990).

[37] A. Bassi, E. Ippoliti and B. Vacchini, On the energy increase in space-collapse models, J. Phys. A 38, 8017 (2005).

[38] S. L. Adler and F. M. Ramazanoğlu, Photon-emission rate from atomic systems in the CSL model, J. Phys. A 40, 13395 (2007).

[39] E. Kreyszig, Introductory functional analysis with applications, Wiley Classics Library, John Wiley \& Sons Inc., New York (1989). 